\title{
Dynamic Kibble-Zurek scaling framework for open dissipative many-body systems crossing quantum transitions
}

\author{
Davide Rossini 1 and Ettore Vicari 10 \\ Dipartimento di Fisica dell'Università di Pisa and Istituto Nazionale di Fisica Nucleare, Largo Pontecorvo 3, I-56127 Pisa, Italy
}

(Received 23 March 2020; accepted 3 May 2020; published 21 May 2020)

\begin{abstract}
We study the quantum dynamics of many-body systems, in the presence of dissipation due to the interaction with the environment, under Kibble-Zurek (KZ) protocols in which one Hamiltonian parameter is slowly, and linearly in time, driven across the critical value of a zero-temperature quantum transition. In particular we address whether, and under which conditions, open quantum systems can develop a universal dynamic scaling regime similar to that emerging in closed systems. We focus on a class of dissipative mechanisms the dynamics of which can be reliably described through a Lindblad master equation governing the time evolution of the system's density matrix. We argue that a dynamic scaling limit exists even in the presence of dissipation, the main features of which are controlled by the universality class of the quantum transition. This requires a particular tuning of the dissipative interactions, the decay rate $u$ of which should scale as $u \sim t_{s}^{-\kappa}$ with increasing the time scale $t_{s}$ of the $\mathrm{KZ}$ protocol, where the exponent $\kappa=z /\left(y_{\mu}+z\right)$ depends on the dynamic exponent $z$ and the renormalizationgroup dimension $y_{\mu}$ of the driving Hamiltonian parameter. Our dynamic scaling arguments are supported by numerical results for $\mathrm{KZ}$ protocols applied to a one-dimensional fermionic wire undergoing a quantum transition in the same universality class of the quantum Ising chain, in the presence of dissipative mechanisms which include local pumping, decay, and dephasing.
\end{abstract}

DOI: 10.1103/PhysRevResearch.2.023211

\section{INTRODUCTION}

The recent experimental progress in the control and manipulation of quantum many-body systems has led to great achievements, opening the door for the realization of quantum simulators [1-6]. However, the effective isolation of a quantum system remains a challenge, since interactions with the environment can have a significant impact in the dynamics, even when they interact weakly. It is thus important to understand the effects of dissipative interactions, irrespective of their strength. This issue is of particular relevance for systems at quantum transitions [7], where the above-mentioned effects are generally relevant, thus tending to suppress the critical quantum correlations $[8,9]$.

Slow passages through quantum transitions allow us to probe some universal features of quantum fluctuations in such circumstances. In this respect, we mention the KibbleZurek (KZ) problem [10-14], related to the amount of final defects, after slow (quasiadiabatic) passages through continuous quantum transitions, from the disorder phase to the order phase. Its scaling predictions have been confirmed by experiments for various physically interesting systems (see, e.g., Refs. [15-20]). KZ-like protocols have been largely employed to investigate the critical dynamics of closed systems,

Published by the American Physical Society under the terms of the Creative Commons Attribution 4.0 International license. Further distribution of this work must maintain attribution to the author(s) and the published article's title, journal citation, and DOI. subject to unitary time evolutions only [21,22]. The open nature of quantum systems, however, may lead to a departure from the dynamic scaling behavior predicted for the isolated case [23-35]. In particular, it has been observed that slower quenches in open systems, or subject to noisy controls, may generate an overabundance of defects when approaching the adiabatic limit in $\mathrm{KZ}$ protocols, sometimes named anti-KZ behavior [36].

Since dissipative mechanisms are expected to give rise to relevant perturbations at the quantum criticality of closed systems $[8,9,37]$ (such as the temperature), they do not generally preserve the universal dynamic properties of quantum transitions. From this point of view, the above-mentioned anti-KZ behavior should not be considered as unexpected. Indeed, due to the general relevance of the perturbations associated with dissipative mechanisms, slower protocols favor the dissipation effects, in that they give them more time to act. Therefore, unlike closed systems, the dynamic behaviors arising from slow changes of the Hamiltonian parameters, across their critical values, do not anymore develop universal critical features controlled by the quantum transition of the closed system. Only an appropriate tuning of the dissipation strength may give rise to a nontrivial interplay with the critical unitary dynamics, developing a dynamic scaling behavior in $\mathrm{KZ}$ protocols controlled by the universality class of the quantum transition.

The issue we address in this paper is whether, and under which conditions, open dissipative systems may still present a universal regime controlled by the universality class of the quantum transition of the closed system. We focus on a class of dissipative mechanisms the dynamics of which can be 
reliably described through a Lindblad master equation [38,39] governing the time evolution of the density matrix of the system [40-42]. We argue that, in the presence of weak dissipation, the dynamics of many-body systems may still develop a scaling behavior under $\mathrm{KZ}$ protocols (i.e., slow changes of one Hamiltonian parameter across its critical value), thus extending the dynamic KZ scaling of closed systems [14]. Its main features, in the presence of weak dissipation, are still controlled by the universality class of the quantum transition, provided the system-environment interaction strength is suitably tuned. This allows us to define a dynamic $\mathrm{KZ}$ scaling limit in the presence of dissipation. In particular, we argue that the decay rate $u$ of the dissipative interactions must scale as a power law $u \sim t_{s}^{-\kappa}$ with increasing the time scale $t_{s}$ of the slow variations of the $\mathrm{KZ}$ protocol, where $\kappa<1$ is an appropriate positive exponent, depending on the universal critical exponents of the quantum transition. The suppression of the dissipation rate is necessary to observe universal dynamic scaling and it is analogous to that found in other dynamic problems with dissipation at quantum transitions $[8,9]$.

To check our general framework, we present a numerical analysis of $\mathrm{KZ}$ protocols applied to the fermionic Kitaev wire [43] across its quantum transition (belonging to the same universality class of the one appearing in quantum Ising chains) in the presence of dissipative mechanisms including local pumping, decay, and dephasing. This model can be exactly and fully solved (i.e., with respect to its full excitation spectrum) even with a large number of sites, up to a few thousands, thus enabling us to perform an accurate numerical investigation of the dynamic KZ scaling behavior put forward. Our results nicely confirm the emerging of a dynamic scaling in the limit of slow passages across the quantum transition and in the presence of weak dissipation.

The paper is organized as follows. In Sec. II we describe our dynamic $\mathrm{KZ}$ protocol and discuss the Lindblad modelization of a dissipative system-environment interaction. In Sec. III we summarize the main features of the dynamic KZ scaling limit, and the emerging scaling laws in the limit of large time scale of the KZ protocol. In Sec. IV we extend the dynamic KZ scaling laws to allow for the presence of dissipation, thus achieving a unique framework to discuss the interplay between (critical) coherent and dissipative drivings. Subsequently we introduce the open-system Kitaev quantum wire, which represents our theoretical laboratory to check the phenomenological dynamic KZ scaling we put forward (Sec. V), and present extensive numerical analyses of $\mathrm{KZ}$ protocols for that model at its zero-temperature quantum transition in the presence of dissipation (Sec. VI). Finally, Sec. VII contains a brief summary and some concluding remarks.

\section{DYNAMIC KZ PROTOCOL IN THE PRESENCE OF DISSIPATION}

We consider a many-body system presenting a quantum transition driven by the Hamiltonian parameters. For simplicity, we assume that the Hamiltonian $\hat{H}$ depends on a single relevant parameter $\mu$, the variation of which drives a quantum transition separating two different quantum phases.
The deviation

$$
\bar{\mu} \equiv \mu-\mu_{c}
$$

quantifies the distance from the critical point, located at $\mu=\mu_{c}$. We also suppose that negative values $\bar{\mu}<0$ correspond to the gapped quantum disordered phase. Quasiadiabatic passages through the quantum transition, slowly varying $\mu$ across $\mu=\mu_{c}$, give rise to peculiar out-of-equilibrium phenomena, such as the one related to the so-called KZ problem [11-13,21,22] addressing the formation of defects when passing through quantum critical points, from the gapped disordered phase to the ordered phase.

A standard KZ protocol would proceed as follows.

(i) One starts from the ground state of the many-body system at $\bar{\mu}_{i}<0$, or alternatively from a statistical state described by the Gibbs distribution $\propto e^{-\hat{H}\left(\bar{\mu}_{i}\right) / k_{B} T}$ at small temperature $T$.

(ii) Then the quantum dynamics is driven by slow variations of the relevant parameter $\bar{\mu}$ associated with the quantum transition, for example, linearly as

$$
\bar{\mu}(t)=t / t_{s},
$$

up to a value $\bar{\mu}_{f}>0$. The parameter $t_{s}>0$ denotes the time scale of the slow variations of the Hamiltonian parameter $\bar{\mu}$. The time evolution is unitary, i.e.,

$$
\frac{\partial \rho}{\partial t}=-\frac{i}{\hbar}[\hat{H}(\bar{\mu}), \rho]
$$

where $\rho(t)$ is the density matrix of the many-body system. Even in the limit of very slow changes, corresponding to $t_{s} \rightarrow \infty$, infinite-volume systems cannot satisfy the adiabatic dynamic condition when passing through the transition point, thus developing out-of-equilibrium behaviors. The resulting evolution of the system is usually investigated by monitoring observables obtained by taking expectation values at fixed time. For example, in the case of lattice spin models, one may consider the magnetization, the two-point function of local operators related to the order parameter, etc.

Here we want to study the effects of weak dissipative mechanisms on the slow dynamics across the quantum transition. Therefore, besides the changes of the Hamiltonian parameters, we suppose that the many-body system is also subject to some interaction with the environment. The time dependence of its density matrix $\rho$ can be reasonably described by the Lindblad master equation [40]

$$
\frac{\partial \rho}{\partial t}=-\frac{i}{\hbar}[\hat{H}(\bar{\mu}), \rho]+u \mathbb{D}[\rho],
$$

where the first term in the right-hand side provides the coherent driving, while the second term accounts for the coupling to the environment, characterized by a global coupling constant $u>0$.

We restrict ourselves to homogeneous dissipation mechanisms, preserving translational invariance. In the case of systems weakly coupled to Markovian baths, the trace-preserving superoperator can be written as a sum of local terms, such as $[38,39]$

$$
\begin{gathered}
\mathbb{D}[\rho]=\sum_{o} \mathbb{D}_{o}[\rho], \\
\mathbb{D}_{o}[\rho]=\hat{L}_{o} \rho \hat{L}_{o}^{\dagger}-\frac{1}{2}\left(\rho \hat{L}_{o}^{\dagger} \hat{L}_{o}+\hat{L}_{o}^{\dagger} \hat{L}_{o} \rho\right),
\end{gathered}
$$


where $\hat{L}_{o}$ is the Lindblad jump operator associated with the local system-bath coupling scheme, and $o$ denotes an appropriate spatial coordinate. In quantum optical implementations, the conditions leading to Eqs. (4)-(6) are typically satisfied [42], therefore this formalism constitutes the standard choice for theoretical investigations of this kind of systems.

In the following we analyze the dynamic scaling behavior arising from dynamic protocols of quantum many-body systems in the presence of weak dissipation, thus evolving according to Eq. (4), when the parameter $\mu$ is slowly varied across its critical value $\mu_{c}$ associated with the quantum transition driven by the Hamiltonian, starting from the gapped disordered phase, analogously to the standard KZ protocol for closed systems.

\section{DYNAMIC KZ SCALING FOR CLOSED QUANTUM SYSTEMS}

Before discussing the effects of dissipation, we recall the main features of the dynamic scaling behavior developed by many-body systems unitarily evolving at quantum transitions $[14,44-46]$, and in particular when they are slowly driven across its quantum transition, according to the $\mathrm{KZ}$ protocol described in Sec. II [see Eqs. (2) and (3)].

\section{A. Homogeneous scaling laws}

At the critical point, the low-energy unitary Hamiltonian dynamics develops long-distance correlations, characterized by a diverging equilibrium length scale $\xi \sim|\bar{\mu}|^{-\nu}$ [where $v=1 / y_{\mu}$ and $y_{\mu}$ is the renormalization-group (RG) dimension of the relevant parameter] and the suppression of the gap (energy difference between the lowest states) $\Delta \sim \xi^{-z}$. The correlation-length exponent $v$ and the dynamic exponent $z$ are the critical exponents associated with the universality class of the quantum transition. The dynamics at continuous quantum transitions develop homogeneous scaling laws [12,14,44-57], even in the presence of interactions with an environment $[8,9,37,58]$.

For example, in the case of instantaneous quenches of closed systems, arising from the instantaneous variation of the Hamiltonian parameter from $\bar{\mu}_{i}$ to $\bar{\mu}$, starting from the ground state at $\bar{\mu}_{i}$, the evolution of a generic observable $B$, such as the expectation value of a local operator $\hat{B}$ (assuming translation invariance), satisfies the homogeneous scaling relation [45]

$$
\begin{aligned}
B\left(\bar{\mu}_{i}, \bar{\mu}, t, L\right) & \equiv\langle\Psi(t)|\hat{B}| \Psi(t)\rangle \\
& \approx b^{-y_{B}} \mathcal{B}\left(\bar{\mu}_{i} b^{y_{\mu}}, \bar{\mu} b^{y_{\mu}}, t b^{-z}, L / b\right) .
\end{aligned}
$$

Here $|\Psi(t)\rangle$ indicates the quantum many-body state after the quench, $b$ is an arbitrary positive parameter, $y_{B}$ is the RG dimension of the operator $\hat{B}, L$ is the size of the system, and $\mathcal{B}$ is a universal scaling function apart from normalizations. Equation (7) is expected to provide the asymptotic power-law behavior in the large- $b$ limit.

The KZ protocol focuses on the opposite quasiadiabatic regime, where the driving parameter $\bar{\mu}$ is slowly varied across the quantum transition, starting from the ground state at a given $\bar{\mu}_{i}<0$ and then changing $\bar{\mu}$ linearly in time, as in Eq. (2) (thus the initial condition $\bar{\mu}_{i}$ corresponds to the initial time $\left.t_{i}=t_{s} \bar{\mu}_{i}\right)$. A phenomenological scaling theory is obtained by assuming the homogeneous scaling law

$$
B\left(\bar{\mu}_{i}, t, t_{s}, L\right) \approx b^{-y_{B}} \mathcal{B}\left(\bar{\mu}_{i} b^{y_{\mu}}, \bar{\mu}(t) b^{y_{\mu}}, t b^{-z}, L / b\right),
$$

where, again, $b$ is an arbitrary positive parameter. Analogous scaling equations can be written down for the fixed-time correlations $G_{A B}$ of two local operators $\hat{A}$ and $\hat{B}$ at a distance $x$. Assuming translation invariance,

$$
\begin{aligned}
G_{A B}\left(x, \bar{\mu}_{i}, t, t_{s}, L\right) & \equiv\left\langle\Psi(t)\left|\hat{A}\left(x_{0}\right) \hat{B}\left(x_{0}+x\right)\right| \Psi(t)\right\rangle \\
& \approx b^{-\varphi} \mathcal{G}\left(x / b, \bar{\mu}_{i} b^{y_{\mu}}, \bar{\mu}(t) b^{y_{\mu}}, t b^{-z}, L / b\right),
\end{aligned}
$$

where $\varphi=y_{A}+y_{B}$ and $y_{A}$ and $y_{B}$ are the RG dimensions of the operators $\hat{A}$ and $\hat{B}$, respectively.

The dynamic $\mathrm{KZ}$ scaling framework can be extended to situations where the initial condition is given by a Gibbs ensemble at temperature $T$, by adding a further dependence on the product $T b^{z}$ in the KZ scaling functions of Eqs. (8a) and $(8 b)$.

\section{B. Dynamic scaling in the infinite-volume limit}

We now concentrate on KZ protocols. To derive a dynamic scaling theory for infinite-volume systems, it is possible to exploit the arbitrariness of the scale parameter $b$ in the general homogeneous power laws (8a) and (8b). To this purpose we set

$$
b=\lambda \equiv t_{s}^{\frac{1}{y \mu+z}},
$$

where $\lambda$ is the length scale associated with the KZ protocol, and take the limit $L / \lambda \rightarrow \infty$ (corresponding to taking the socalled thermodynamic limit). This leads to the dynamic KZ scaling ansatz

$$
\begin{aligned}
B\left(\bar{\mu}_{i}, t, t_{s}\right) & \approx \lambda^{-y_{B}} \mathcal{B}_{i}\left(\bar{\mu}_{i} \lambda^{y_{\mu}}, \tau\right), \\
G_{A B}\left(x, \bar{\mu}_{i}, t, t_{s}\right) & \approx \lambda^{-\varphi} \mathcal{G}_{i}\left(x / \lambda, \bar{\mu}_{i} \lambda^{y_{\mu}}, \tau\right),
\end{aligned}
$$

where $\tau$ is the rescaled time:

$$
\tau \equiv t / t_{s}^{\kappa}, \quad \kappa=\frac{z}{y_{\mu}+z} .
$$

The dynamic KZ scaling limit, where the above asymptotic behaviors apply, is obtained by taking $t_{s} \rightarrow \infty$ keeping the arguments of the dynamic scaling functions $\mathcal{B}_{i}$ and $\mathcal{G}_{i}$ fixed. Actually, introducing a time scaling variable related to initial time of the $\mathrm{KZ}$ protocol,

$$
\tau_{i} \equiv t_{i} / t_{s}^{\kappa}, \quad t_{i}=\bar{\mu}_{i} t_{s},
$$

we may rewrite the scaling Eqs. (10a) and (10b) as

$$
\begin{aligned}
B\left(\bar{\mu}_{i}, t, t_{s}\right) & \approx \lambda^{-y_{B}} \widetilde{\mathcal{B}}_{i}\left(\tau_{i}, \tau\right), \\
G_{A B}\left(x, \bar{\mu}_{i}, t, t_{s}\right) & \approx \lambda^{-\varphi} \widetilde{\mathcal{G}}_{i}\left(x / \lambda, \tau_{i}, \tau\right) .
\end{aligned}
$$

Note that the scaling functions $\mathcal{B}_{i}, \mathcal{G}_{i}$ and $\widetilde{\mathcal{B}}_{i}, \widetilde{\mathcal{G}}_{i}$ in Eqs. (10a)(10b) and (13a)-(13b) do not coincide, but are trivially related by the change of scaling variables.

Since the KZ protocol starts from $\bar{\mu}_{i}<0$ corresponding to the gapped phase, where the gap decreases as $\Delta \sim \xi^{-z}$ and 
the ground-state length scale $\xi$ diverges only at the critical point $\bar{\mu}=0$, the emerging dynamic KZ scaling should be independent of the actual finite value of $\bar{\mu}_{i}<0$, if this is kept fixed in the dynamic KZ scaling limit. This is essentially due to the fact that, in a gapped phase, the evolution arising from slow changes of the parameters is essentially adiabatic, from $\bar{\mu}_{i}$ to the relevant scaling interval $\delta_{\mu}$ around $\bar{\mu}=0$, which effectively decreases as

$$
\delta_{\bar{\mu}} \sim t_{s}^{-1+\kappa} \rightarrow 0
$$

in the dynamic KZ scaling limit. Therefore, when increasing $t_{s}$, keeping $\mu_{i}<0$ constant and finite, the dynamic KZ scaling must be independent of $\bar{\mu}_{i}$, corresponding to the $\tau_{i} \rightarrow-\infty$ limit of the relations (13a) and (13b). Therefore this leads to the dynamic scaling ansatz

$$
\begin{aligned}
B\left(\bar{\mu}_{i}, t, t_{s}\right) & \approx \lambda^{-y_{B}} \mathcal{B}_{\infty}(\tau), \\
G_{A B}\left(x, \bar{\mu}_{i}, t, t_{s}\right) & \approx \lambda^{-\varphi} \mathcal{G}_{\infty}(x / \lambda, \tau) .
\end{aligned}
$$

The dynamic scaling functions introduced above are expected to be universal with respect to changes of the microscopic details of the Hamiltonian within the given universality class. Of course, like any scaling function at quantum transitions, such a universality holds apart from a multiplicative overall constant and normalizations of the scaling variables. The approach to the asymptotic dynamic scaling behavior is expected to be generally characterized by power-law suppressed corrections.

We finally mention that the so-called KZ problem genuinely addresses the formation of defects when slowly crossing the quantum transition, from the disordered to the ordered phase. The above scaling arguments in the dynamic KZ limit (see Refs. [11,14,22]) lead to the expectation that the density of defects arising after crossing the transition scales as the inverse scaling volume $\lambda^{-d}$ [see Eq. (9)], that is

$$
\rho_{\text {defects }} \sim \lambda^{-d}=t_{s}^{-\frac{d}{y_{\mu}+z}} .
$$

This scaling behavior has been verified in experiments (see, e.g., Refs. [15-20,36,59,60]).

\section{Dynamic finite-size scaling}

The scaling Eqs. (8a) and (8b) also allow us to derive dynamic finite-size scaling (FSS) relations, which are valid far from the thermodynamic limit, and which extend those predicted by the FSS theory for systems at equilibrium [61-63]. For example, by setting $b=L$ in Eq. (8b), we obtain

$$
G_{A B}\left(x, \bar{\mu}_{i}, t, t_{s}, L\right) \approx L^{-\varphi} \mathcal{G}_{L}\left(x / L, \bar{\mu}_{i} L^{y_{\mu}}, \bar{\mu}(t) L^{y_{\mu}}, t L^{-z}\right) .
$$

This dynamic FSS behavior is expected to be obtained by taking $L \rightarrow \infty$, while keeping the arguments of the scaling function $\mathcal{G}_{L}$ fixed. One may introduce more convenient scaling variables, which are combinations of those entering Eq. (17). For example, one can write it as

$$
G_{A B}\left(x, \bar{\mu}_{i}, t, t_{s}, L\right) \approx L^{-\varphi} \mathcal{G}_{L}\left(x / L, \tau_{i}, \tau, v\right),
$$

where

$$
v \equiv t_{s} / L^{y_{\mu}+z}
$$

and $\tau$ and $\tau_{i}$ are defined in Eqs. (11) and (12), respectively.
Assuming again that the $\mathrm{KZ}$ protocol starts from the gapped disordered phase and the initial $\bar{\mu}_{i}<0$ is kept fixed in the dynamic scaling limit, the same dynamic FSS is expected to hold, irrespective of the value of $\bar{\mu}_{i}$. Thus, the dynamic FSS in Eq. (18) simplifies into

$$
G_{A B}\left(x, \bar{\mu}_{i}, t, t_{s}, L\right) \approx L^{-\varphi} \mathcal{G}_{L, \infty}(x / L, \tau, v) .
$$

Indeed, with increasing $L$, the dynamic FSS occurs within a smaller and smaller interval of values of $|\mu|$ around $\bar{\mu}=0$ : since the time interval of the dynamic process scales as $t_{\mathrm{sca}} \sim t_{s}^{\kappa}$, the relevant interval of values of $|\bar{\mu}|$ shrinks as $t_{\mathrm{sca}} / t_{s} \sim L^{-y_{\mu}}$, when keeping $v$ fixed.

Note that, in the limit $v \rightarrow \infty$, the evolution as a function of $\bar{\mu}(t)=t / t_{s}$ corresponds to an adiabatic dynamics. Indeed, since the finite-size $L$ guarantees the presence of a gap between the lowest states, one may adiabatically cross the critical point in the limit $v \rightarrow \infty$, passing through the ground states of the finite-size system for $\bar{\mu}(t)$. The adiabatic evolution across the transition point is prevented only when $L \rightarrow \infty$ (before the limit $t_{s} \rightarrow \infty$ ), i.e., when the time scale of the critical correlations diverges, since $\tau_{\mathrm{cr}} \sim \Delta^{-1} \sim L^{z}$.

\section{DYNAMIC KZ SCALING FOR OPEN QUANTUM SYSTEMS}

\section{A. Dynamic scaling allowing for dissipation}

In this section, we extend the dynamic scaling theory outlined in Sec. III to systems subject to dissipative interactions with the environment, so that the time dependence of the density matrix $\rho$ is described by the Lindblad master equation (4). Namely, we assume that the quantum evolution arising from the $\mathrm{KZ}$ protocol occurs in the presence of dissipation with the effective coupling $u>0$, thus being ruled by Eq. (4). The resulting dynamic KZ scaling framework will provide a unique framework to discuss the interplay between (critical) coherent and dissipative drivings.

The dynamic behavior in the presence of weak dissipation has been addressed within a phenomenological dynamic scaling theory in Refs. [8,9], extending the dynamic scaling scenario holding for closed systems. This has been obtained by adding a further dependence associated with the dissipation parameter $u$ in the dynamic scaling relations (8a) and (8b), through a power law $u b^{z}$, where the dynamic exponent $z$ ensures the substantial balance (i.e., competition) with the critical coherent driving. We recall that this hypothesis has been put forward after noting that the parameter $u$ of the dissipator in Eq. (4) plays the role of a decay rate, i.e., of an inverse relaxation time, of the associated dissipative process [40]. Thus, to observe a nontrivial competition between critical coherent dynamics and dissipation, the dissipative coupling must be comparable to the gap of the critical Hamiltonian, therefore its scaling variable must be controlled by the dynamic exponent $z$.

Following the above reasoning, we conjecture that $\mathrm{KZ}$ protocols in the presence of dissipation develop homogeneous 
laws, such as

$$
\begin{aligned}
& B\left(\bar{\mu}_{i}, t, t_{s}, u, L\right) \\
& \quad \equiv \operatorname{Tr}[\rho(t) \hat{B}] \\
& \quad \approx b^{-y_{B}} \mathcal{B}\left(\bar{\mu}_{i} b^{y_{\mu}}, \bar{\mu}(t) b^{y_{\mu}}, t b^{-z}, L / b, u b^{z}\right),
\end{aligned}
$$

and

$$
\begin{aligned}
& G_{A B}\left(x, \bar{\mu}_{i}, t, t_{s}, u, L\right) \\
& \quad \equiv \operatorname{Tr}[\rho(t) \hat{A} \hat{B}] \\
& \quad \approx b^{-\varphi} \mathcal{G}\left(x / b, \bar{\mu}_{i} b^{y_{\mu}}, \bar{\mu}(t) b^{y_{\mu}}, t b^{-z}, L / b, u b^{z}\right),
\end{aligned}
$$

similar to those in Eqs. (8a) and (8b), but with one additional scaling variable associated with $u$.

\section{B. Dynamic scaling in the infinite-volume limit}

Analogously to the dynamics of closed systems, it is possible to derive scaling laws in the thermodynamic limit, by fixing $b$ as in Eq. (9) and taking $L / \lambda \rightarrow \infty$. One can easily show that Eqs. (21a) and (21b) imply the dynamic KZ scaling ansatz

$$
\begin{aligned}
B\left(\bar{\mu}_{i}, t, t_{s}, u\right) & \approx \lambda^{-y_{B}} \mathcal{B}_{i}\left(\tau_{i}, \tau, \gamma\right), \\
G_{A B}\left(x, \bar{\mu}_{i}, t, t_{s}, u\right) & \approx \lambda^{-\varphi} \mathcal{G}_{i}\left(x / \lambda, \tau_{i}, \tau, \gamma\right),
\end{aligned}
$$

where we introduced the scaling variable $\gamma$ associated with the dissipation parameter:

$$
\gamma=u t_{s}^{\kappa}, \quad \kappa=\frac{z}{y_{\mu}+z} .
$$

The above scaling laws are expected to provide the asymptotic behavior in the $t_{s} \rightarrow \infty$ limit while keeping the scaling variables fixed, including $\gamma$. In the limit $\gamma \rightarrow 0$, we must recover the scaling laws of the closed systems subject to unitary evolutions only. More importantly, the above scaling laws tell us that the dissipation effects are expected to be negligible when $u \ll t_{s}^{-\kappa}$.

Note that, like for closed systems, the large- $t_{s}$ limit of $\mathrm{KZ}$ protocols starting from finite and fixed $\bar{\mu}_{i}<0$ should correspond to the limit $\tau_{i} \rightarrow-\infty$ in the right-hand side of Eqs. (22a) and (22b). Indeed, the dissipation with coupling strength $u \sim \lambda^{-z}$ is not expected to play any relevant role at finite $\bar{\mu}_{i}<0$, where the gap is $\Delta=O(1)$, while it should compete with the unitary evolution only very close to $\bar{\mu}=0$ where $u \sim \Delta \sim \lambda^{-z}$. Therefore, under such conditions we expect the scaling behavior

$$
\begin{aligned}
B\left(\bar{\mu}_{i}, t, t_{s}, u\right) & \approx \lambda^{-y_{B}} \mathcal{B}_{\infty}(\tau, \gamma), \\
G_{A B}\left(x, \bar{\mu}_{i}, t, t_{s}, u\right) & \approx \lambda^{-\varphi} \mathcal{G}_{\infty}(x / \lambda, \tau, \gamma) .
\end{aligned}
$$

We mention that, in the above $\mathrm{KZ}$ scaling limit allowing for dissipation, the scaling law associated with the number of defects [see Eq. (16)] should be replaced with

$$
\rho_{\text {defects }} \approx \lambda^{-d} \mathcal{D}(\gamma)=t_{s}^{-\frac{d}{y_{\mu}+z}} \mathcal{D}(\gamma),
$$

where the dependence on the dissipative coupling $u$ enters the scaling function $\mathcal{D}$ through the scaling variable $\gamma$. Of course, one must recover the scaling law (16) for $\gamma=0$. Notice that Eq. (25) is expected to apply when the arrival phase is gapped, or, generally, when the final value $\bar{\mu}_{f}$ of $\bar{\mu}$ scales appropriately to $\bar{\mu}=0$, i.e., as $\bar{\mu}_{f} \sim t_{s}^{-1+\kappa}$ when increasing $t_{s}$.

\section{Dynamic finite-size scaling}

The dynamic FSS behavior can be obtained by setting $b=L$ in Eqs. (21a) and (21b), thus extending the results contained in Sec. III C to allow for the dissipation term of the Lindblad equation. Namely,

$$
G_{A B}\left(x, \bar{\mu}_{i}, t, t_{s}, u, L\right) \approx L^{-\varphi} \mathcal{G}_{L}\left(x / L, \tau_{i}, \tau, v, \gamma_{L}\right),
$$

where

$$
\gamma_{L}=u L^{z}
$$

The above scaling law can be obtained in the $L \rightarrow \infty$ limit while keeping the scaling variables fixed, including $\gamma_{L}$.

Moreover, assuming again that the quantum phase for $\bar{\mu}<0$ is gapped, with $\Delta \sim \xi^{-z}$, and the ground-state length scale $\xi$ diverges only at the critical point $\bar{\mu}=0, \mathrm{KZ}$ protocols associated with any finite initial $\bar{\mu}_{i}<0$ develop the same dynamic FSS independently of their actual values. Thus, the dynamic FSS can be written as

$$
G_{A B}\left(x, \bar{\mu}_{i}, t, t_{s}, u, L\right) \approx L^{-\varphi} \mathcal{G}_{L, \infty}\left(x / L, \tau, v, \gamma_{L}\right) .
$$

Indeed, similarly to the infinite-volume case, the dissipation with coupling strength $u \sim L^{-z}$ is not expected to play any relevant role at finite $\bar{\mu}_{i}<0$, where the gap is $\Delta=O(1)$, while it should compete with the unitary evolution only very close to the critical point where $u \sim \Delta \sim L^{-z}$. Like for closed systems, the dynamic scaling limit thus involves smaller and smaller intervals of values of $|\bar{\mu}|$ around $\bar{\mu}=0$ with increasing $L$ : since the time interval of the dynamic process scales as $t_{\text {sca }} \sim t_{s}^{\kappa}$, the relevant interval of values of $|\mu|$ shrinks as $t_{\text {sca }} / t_{s} \sim L^{-y_{\mu}}$, when keeping $v$ fixed.

\section{KZ PROTOCOLS FOR THE KITAEV QUANTUM WIRE SUBJECT TO DISSIPATION}

To verify the dynamic KZ scaling laws put forward in the previous sections, and in particular in Sec. IV, we consider a Kitaev quantum wire defined by the Hamiltonian [43]

$$
\hat{H}_{\mathrm{K}}=-J \sum_{j=1}^{L}\left(\hat{c}_{j}^{\dagger} \hat{c}_{j+1}+\delta \hat{c}_{j}^{\dagger} \hat{c}_{j+1}^{\dagger}+\text { H.c. }\right)-\mu \sum_{j=1}^{L} \hat{n}_{j},
$$

where $\hat{c}_{j}$ is the fermionic annihilation operator on the $j$ th site of the chain, $\hat{n}_{j} \equiv \hat{c}_{j}^{\dagger} \hat{c}_{j}$ is the density operator, and $\delta>0$. We set $\hbar=1$, and $J=1$ as the energy scale. Moreover we fix $\delta=1$. We consider antiperiodic boundary conditions, $\hat{c}_{L+1}=-\hat{c}_{1}$, and even $L$ for computational convenience.

The fermionic system described by the Hamiltonian (29) undergoes a continuous quantum transition at $\mu=\mu_{c}=-2$, independently of $\delta$, belonging to the same universality class of that of the quantum Ising chain (when $\delta>0$ ), i.e., the two-dimensional Ising universality class $[7,43]$, characterized by the length-scale critical exponent $v=1$, related to the RG dimension $y_{\mu}=1 / \nu=1$ of the Hamiltonian parameter $\mu$ (more precisely of the difference $\bar{\mu} \equiv \mu-\mu_{c}$ ). The dynamic 
exponent associated with the unitary quantum dynamics is $z=1$. Moreover, the RG dimension of the fermionic operators $\hat{c}_{j}$ and $\hat{c}_{j}^{\dagger}$ is $y_{\hat{c}}=y_{\hat{c}^{\dagger}}=1 / 2$, and that of the density operator $\hat{n}_{j}$ is $y_{\hat{n}}=1$ [7]. Details on the correspondence with the quantum Ising chain are provided in Appendix.

We focus on the dynamic behavior of the Fermi lattice gas (29) close to its quantum transition, in the presence of homogeneous dissipation mechanisms following the Lindblad equation (4). The dissipator $\mathbb{D}[\rho]$ is defined as a sum of local (single-site) terms of the form

$$
\mathbb{D}_{j}[\rho]=\hat{L}_{j} \rho \hat{L}_{j}^{\dagger}-\frac{1}{2}\left(\rho \hat{L}_{j}^{\dagger} \hat{L}_{j}+\hat{L}_{j}^{\dagger} \hat{L}_{j} \rho\right),
$$

where $\hat{L}_{j}$ denotes the Lindblad jump operator associated with the system-bath coupling scheme, and the index $j$ corresponds to a lattice site [thus replacing the index $o$ in Eqs. (5) and (6)]. The onsite Lindblad operators $\hat{L}_{j}$ describe the coupling of each site with an independent bath. We consider dissipation mechanisms associated with either particle losses (1), pumping (p), or dephasing (d), respectively [8,29,64-68]:

$$
\hat{L}_{\mathrm{l}, j}=\hat{c}_{j}, \quad \hat{L}_{\mathrm{p}, j}=\hat{c}_{j}^{\dagger}, \quad \hat{L}_{\mathrm{d}, j}=\hat{n}_{j} .
$$

The choice of such dissipators turns out to be particularly convenient for the numerical analysis, allowing us to scale the difficulty of the problem linearly with $L$ and thus to obtain results for the Kitaev wire with thousands of sites (see Sec. VI). This is important, in view of the necessity to perform adequate numerical checks of a new scaling theory lying on phenomenological grounds.

The $\mathrm{KZ}$ protocol that we consider starts from the ground state of $\hat{H}_{K}$ for a generic $\bar{\mu}_{i}<0$, where the system is gapped, $\Delta=|\bar{\mu}|+O\left(L^{-2}\right)$, while $\Delta \sim L^{-1}$ at $\bar{\mu}=0$ (see Appendix). Then the system evolves according to Eq. (4) with a timedependent parameter $\bar{\mu}(t)=t / t_{s}$, starting from $t_{i}<0$ such that $\bar{\mu}_{i}=t_{i} / t_{s}$. To characterize the dynamic properties of the evolution described by the Lindblad equation, and in particular the corresponding asymptotic large-time behavior, we consider the fixed-time correlations

$$
\begin{aligned}
& P(x, t)=\operatorname{Tr}\left[\rho(t)\left(\hat{c}_{j}^{\dagger} \hat{c}_{j+x}^{\dagger}+\hat{c}_{j+x} \hat{c}_{j}\right)\right], \\
& C(x, t)=\operatorname{Tr}\left[\rho(t)\left(\hat{c}_{j}^{\dagger} \hat{c}_{j+x}+\hat{c}_{j+x}^{\dagger} \hat{c}_{j}\right)\right] \\
& G(x, t)=\operatorname{Tr}\left[\rho(t) \hat{n}_{j} \hat{n}_{j+x}\right]-\operatorname{Tr}\left[\rho(t) \hat{n}_{j}\right] \operatorname{Tr}\left[\rho(t) \hat{n}_{j+x}\right],
\end{aligned}
$$

where $j, x \in[1, L / 2]$.

The dynamic KZ scaling of the above correlation functions is expected to be given by the general scaling laws reported for the generic two-point function $G_{A B}$ in Sec. IV, taking into account that $\varphi=1$ for the correlations $P$ and $C$ (since $y_{\hat{c}}=y_{\hat{c}^{\dagger}}=1 / 2$ ), while $\varphi=2$ for $G$ (since $\left.y_{\hat{n}}=1\right)$. This scaling scenario should hold for all the considered dissipation mechanisms [see Eq. (31)]. Of course, the corresponding scaling functions are expected to differ.

\section{NUMERICAL RESULTS}

We now present the results of a series of numerical computations we have performed on the Kitaev quantum wire. As stated above, this model is amenable to a direct solvability for systems with $O\left(10^{3}\right)$ sites, thus representing the ideal playground for open quantum lattice problems, given the remarkable difficulty to simulate the dynamics of interacting many-body quantum systems coupled to an external bath.

For the specific choice of dissipators in Eq. (31), the exponential complexity of the Kitaev chain can be semianalytically reduced to a polynomial one $[8,29,64,69]$. In particular, in the presence of particle losses or pumping and for translationally invariant systems, the driven-dissipative quantum dynamics ruled by the master equation (4) can be exactly solved by decoupling in Fourier space the various sectors with different momenta, analogously to fermionic Gaussian Hamiltonian models. Similar strategies can be adopted for more general inhomogeneous (disordered) lossy dynamics, provided the Liouvillian operator remains quadratic in the creation and annihilation operators for fermions. On the other hand, although the quantum dynamics with a dephasing mechanism cannot be simply obtained, two-point observables are still fully captured by a set of coupled linear differential equations, the number of which increases linearly with the number of sites $L$ (see, e.g., the Appendix in Ref. [8] for details). The latter can be integrated, e.g., with a standard fourth-order Runge-Kutta method.

\section{A. Dynamic KZ scaling in the infinite-volume limit}

We first discuss systems in the thermodynamic limit. To ensure that finite-size corrections are negligible on the scale of all the numerics presented below for the dynamic scaling, we have carefully checked that (in all cases treated here) systems of size $L=2^{12}=4096$ allow us to simulate $\mathrm{KZ}$ protocols with a length scale $\lambda$ up to $O\left(10^{2}\right)$, corresponding to time scales

$$
t_{s}=\lambda^{y_{\mu}+z}=\lambda^{2},
$$

of the order $O\left(10^{4}\right)$ [see Eq. (2)].

\section{Unitary $\mathrm{KZ}$ dynamics}

Before discussing the effects of dissipation, it is instructive to present the outcomes of a typical $\mathrm{KZ}$ protocol for the unitary dynamics of the Kitaev quantum wire, without dissipation $(u=0)$. Figure 1 shows the time behavior of the fixed-time correlation $P(x, t)$ at fixed $x / \lambda$ [see Eq. (32a)] during a $\mathrm{KZ}$ protocol starting from a fixed rescaled initial time $\tau_{i}<0$ (in the figure, $\tau_{i}=-10$ ) and running up to positive values of the rescaled time. In this way, the Hamiltonian parameter $\bar{\mu} \equiv \mu-\mu_{c}$ of $\hat{H}_{K}$ is slowly changed in time starting from an initial value $\bar{\mu}_{i}=\tau_{i} / \lambda<0$, the absolute value of which decreases with the $\mathrm{KZ}$ length scale $\lambda$, through the critical point $\bar{\mu}=0$ at $t=\tau=0$. The various parameters have been rescaled according to Eq. (13b): we set $\varphi=1$ and $\kappa=1 / 2$, and we plot $\lambda P(x, t)$ as a function of $\tau$, for increasing values of $\lambda$, while keeping the scaling variables $x / \lambda$ and $\tau_{i}$ constant. This corresponds to a progressive increase of the time scale $t_{s}$ of the slow variations of the Hamiltonian parameter $\bar{\mu}$, according to Eq. (33). In Fig. 1 we show results for the rescaled distance $x / \lambda=1$ only; other values of $x / \lambda$ present analogous behaviors. Already for values of $\lambda \sim 10$, the curves approach a nontrivial scaling behavior, in accordance with the general KZ scaling theory for closed systems (see also the zoom in the upper right inset). The oscillating behavior for $\tau>0$ is likely due to adiabaticity losses, which are ascribable 


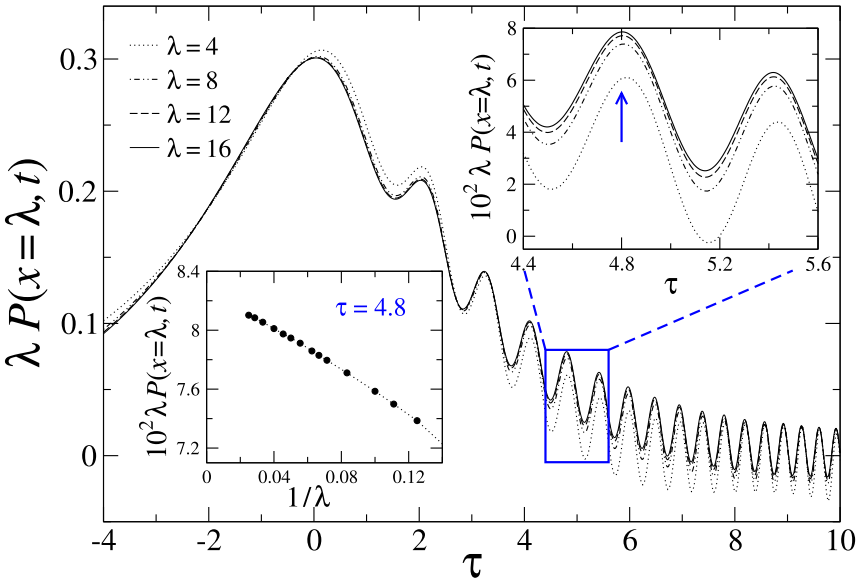

FIG. 1. Rescaled correlation $\lambda P(x, t)$, fixing $x / \lambda=1$, for the unitary dynamics of the Kitaev quantum wire in the thermodynamic limit, as a function of the scaling time variable $\tau$. Here we fix the scaling variable associated with the initial time, $\tau_{i}=-10$. Lines with different styles are for various values of the length scale $\lambda$, from 4 to 16 , as indicated in the legend; these correspond to increasing the time scales as $t_{s}=\lambda^{2}$. The upper right inset shows a magnification of the data for $4.4<\tau<5.6$, while the lower left inset displays rescaled correlations as a function of $1 / \lambda$, for fixed $\tau=4.8$ (arrow in the upper inset), supporting an $O\left(\lambda^{-1}\right)$ approach to the asymptotic $\mathrm{KZ}$ scaling limit. Analogous results are obtained for other values of the scaling variables $x / \lambda$ and $\tau_{i}$, and for the correlations $C(x, t)$ and $G(x, t)$.

to the gapless point at $\tau=0$. The approach to the asymptotic behavior in the limit $\lambda \rightarrow \infty$ is analyzed in the lower inset, for fixed $\tau=4.8$, where we collected data up to $\lambda=40$ (corresponding to time scales up to $t_{s}=1600$ ). As expected, corrections are suppressed with a power-law behavior that is compatible with $O(1 / \lambda)$.

As stated in the previous sections, the dynamic KZ scaling is also expected to be independent of the actual value of $\bar{\mu}_{i}$, if this is kept fixed in the dynamic KZ limit. A numerical verification of this conjecture is presented in Fig. 2, for a situation similar to that in Fig. 1 but fixing $\bar{\mu}_{i}<0$, rather than $\tau_{i}<0$. Specifically, we have analyzed the three correlation functions $P(x, t), C(x, t)$, and $G(x, t)$ [see Eqs. (32a)-(32c)] along a KZ protocol where we fixed the initial condition $\bar{\mu}_{i}$. Even in this case we can see that, after properly rescaling the various parameters and observables, the curves nicely approach a scaling behavior, which appears to be independent of the choice of $\bar{\mu}_{i}$. As previously discussed, the critical point located at $\bar{\mu}=0$ prevents the system from remaining in the instantaneous ground state.

A more accurate analysis of the independence of the dynamic scaling functions from the initial condition $\bar{\mu}_{i}$ is provided in the three insets (each for a different correlation function), where we spotlight the convergence of the rescaled observables with $\lambda \rightarrow \infty$, for a fixed value of $\tau$. In all the three cases we observe that the extrapolated asymptotic value seems to be independent of the two specific $\bar{\mu}_{i}$ analyzed. Notice, however, that, while for the red data sets $\left(\bar{\mu}_{i}=-0.5\right)$ the convergence to the asymptotic behavior appears regular and compatible with a power law $\sim \lambda^{-1}$, the black data sets
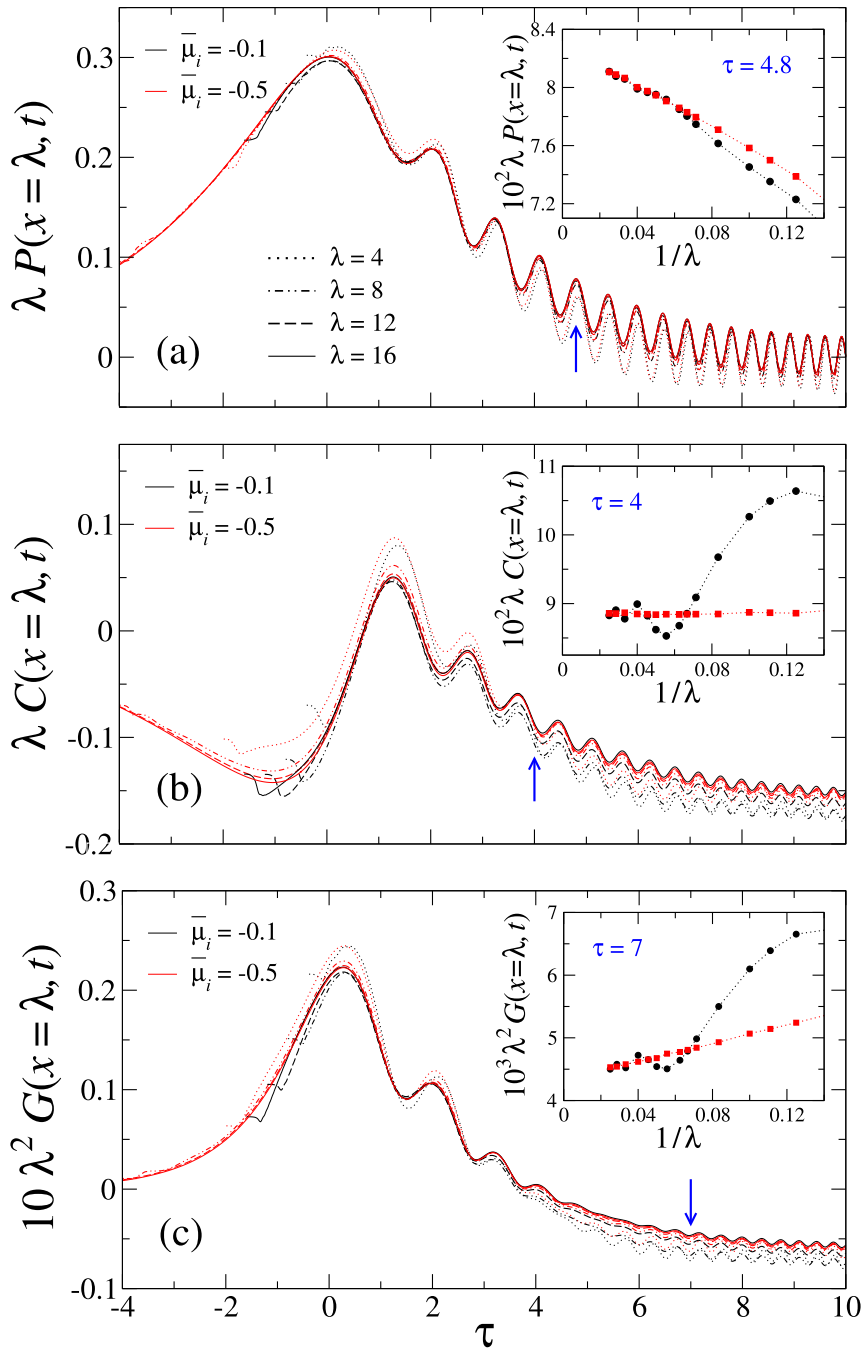

FIG. 2. Rescaled correlations $\lambda P(x, t)$ (a), $\lambda C(x, t)$ (b), and $\lambda^{2} G(x, t)$ (c), at fixed $x / \lambda=1$ (results for other values of $x / \lambda$ show analogous behaviors), for the unitary dynamics of the Kitaev quantum wire in the thermodynamic limit, as a function of the scaling variable $\tau$. Different line styles stand for various values of the length scale $\lambda$, from 4 to 16 , analogously to Fig. 1 (see legend). Data belonging to one of the two color sets correspond to a given initial Hamiltonian parameter $\bar{\mu}_{i}<0$, which is kept fixed and equal to either $\bar{\mu}_{i}=-0.1$ (black circles) or $\bar{\mu}_{i}=-0.5$ (red squares). The insets in the three panels display rescaled correlations as a function of $1 / \lambda$, for both cases of $\bar{\mu}_{i}$ presented in the main frames, at the $\tau$ value indicated by the blue arrow.

$\left(\bar{\mu}_{i}=-0.1\right)$ [especially for $C(x, t)$ and $\left.G(x, t)\right]$ exhibit oscillations in $1 / \lambda$ (at least up to $\lambda=40$ ), which should be ascribed to the proximity of the initial ground state at $\bar{\mu}_{i}$ with that at the critical point $\bar{\mu}=0$. We have verified that the above observations hold also for other values of $\tau$ and for different initial conditions $\bar{\mu}_{i}$ (not shown), with a faster convergence for larger values of $\left|\bar{\mu}_{i}\right|$.

\section{Dissipative KZ dynamics}

We now turn to a situation where the Hamiltonian Kitaev chain $\hat{H}_{K}$ is coupled to a Markovian bath in the form of either incoherent particle losses, pumping, or dephasing [the three 

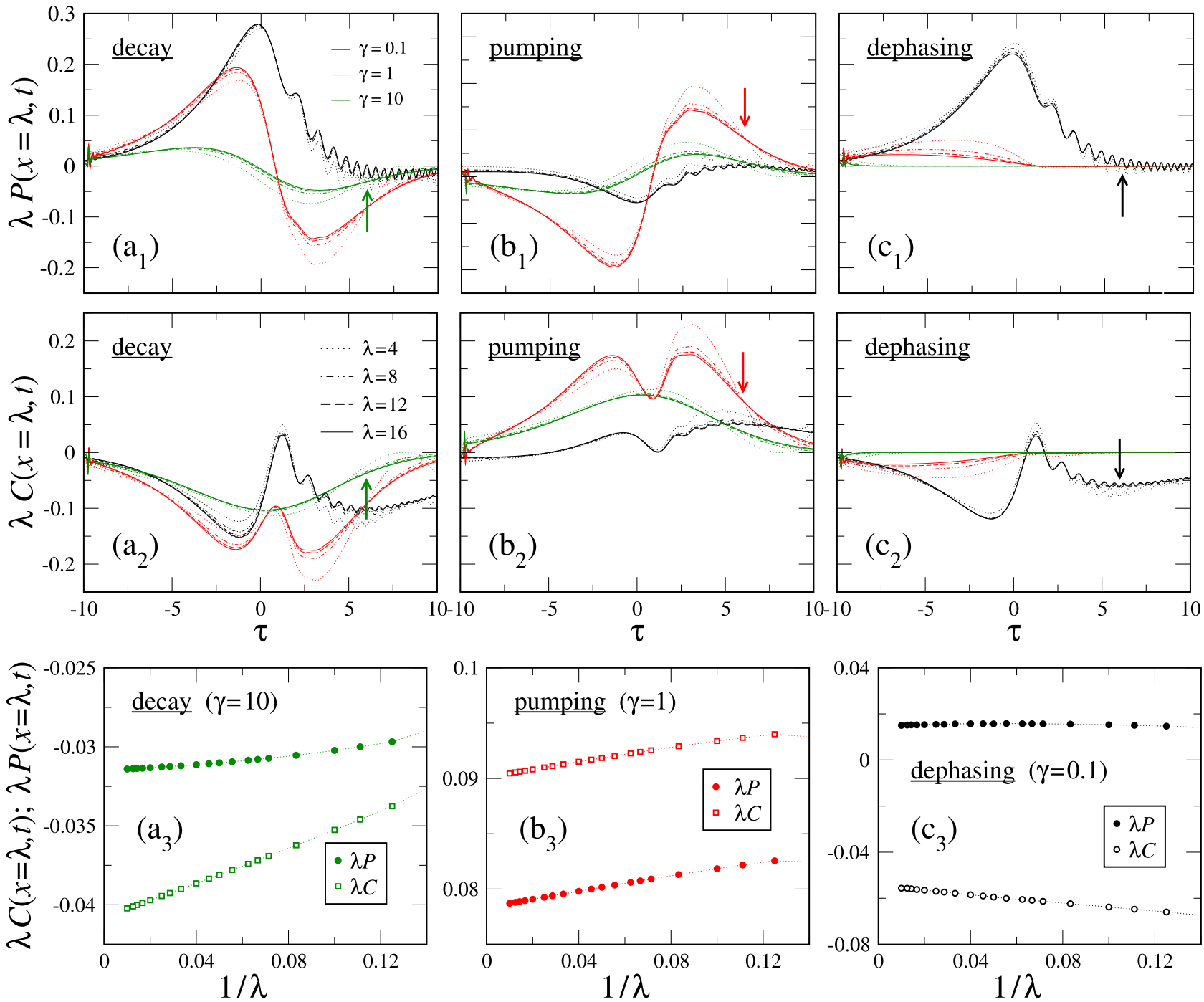

FIG. 3. Rescaled correlations $\lambda P(x, t)\left[\left(\mathrm{a}_{1}\right),\left(\mathrm{b}_{1}\right),\left(\mathrm{c}_{1}\right)\right]$ and $\lambda C(x, t)\left[\left(\mathrm{a}_{2}\right),\left(\mathrm{b}_{2}\right),\left(\mathrm{c}_{2}\right)\right]$, at fixed rescaled distance $x / \lambda=1$ and initial rescaled time $\tau_{i}=-10$, for the dissipative Kitaev quantum wire in the thermodynamic limit, as a function of the scaling variable $\tau$. Analogous scaling behaviors are observed for other values of $x / \lambda$ and $\tau_{i}$, with different asymptotic scaling functions of $\tau$. Each panel in one of the three columns refers to a specific type of dissipation mechanism [see Eq. (31)]: decay $\left[\left(\mathrm{a}_{1}\right),\left(\mathrm{a}_{2}\right),\left(\mathrm{a}_{3}\right)\right]$, pumping $\left[\left(\mathrm{b}_{1}\right),\left(\mathrm{b}_{2}\right),\left(\mathrm{b}_{3}\right)\right]$, and dephasing $\left[\left(\mathrm{c}_{1}\right),\left(\mathrm{c}_{2}\right)\right.$, $\left(c_{3}\right)$ ]. The color code stands for three rescaled dissipative couplings: $\gamma=0.1$ (black), $\gamma=1$ (red), and $\gamma=10$ (green). Different line styles are for various values of $\lambda$, from 8 to 16 (see legend). $\left(a_{3}\right),\left(b_{3}\right),\left(c_{3}\right)$ Rescaled correlations as a function of $1 / \lambda$, up to $\lambda=10^{2}$, for $\tau=6$ (arrows in the panels above) and a given value of $\gamma$ for each panel (see figure).

different types of Lindblad operators are reported in Eq. (31) and are supposed to act uniformly over all the sites of the chain]. According to the dynamic KZ scaling framework discussed in Sec. IV, an additional scaling variable $\gamma$ associated with the dissipation strength $u$ needs to be considered [see Eq. (23)]. In passing we note that the dissipation parameter $u=\gamma / \lambda$ entering the master equation (4) is inversely proportional to $\lambda$, therefore it needs to be progressively decreased down to zero when increasing the KZ length scale $\lambda$. For the two-point correlations analyzed here, one thus expects the emerging scaling behavior (22b).

We proceed as in Sec. VIA 1 in the absence of dissipation, and first address $\mathrm{KZ}$ protocols where the Hamiltonian parameter $\bar{\mu}$ is slowly increased and driven across a critical point $(\bar{\mu}=0)$, starting from an initial value $\bar{\mu}_{i}<0$ such that the corresponding value of the rescaled time $\tau_{i}$ is kept fixed. Results for the correlations $P(x, t)$ and $C(x, t)$, in the presence of either decay, pumping, or dephasing, are shown in Fig. 3 [analogous outcomes have been obtained for $G(x, t)$-not shown]. Again, we present results for the particular rescaled distance $x / \lambda=1$ and rescaled initial time $\tau_{i}=-10$; analogous scaling behaviors are observed for other values of $x / \lambda$ and $\tau_{i}$, but, of course, with different asymptotic scaling functions of $\tau$.

Figures 3( $\left.a_{1}\right), 3\left(b_{1}\right), 3\left(c_{1}\right), 3\left(a_{2}\right), 3\left(b_{2}\right)$, and 3( $\left.c_{2}\right)$ evidence that, after a proper rescaling of the various variables, and in particular fixing the rescaled dissipation rate $\gamma$ as in Eq. (23), the two observables nicely approach a scaling function with increasing $\lambda$, that is, with increasing the KZ time scale $t_{s}=\lambda^{2}$ [see Eq. (33)]. Of course, the latter function depends both on the type of dissipation and on $\gamma$. In particular, numerical data show that the dephasing mechanism appears to be more effec- 

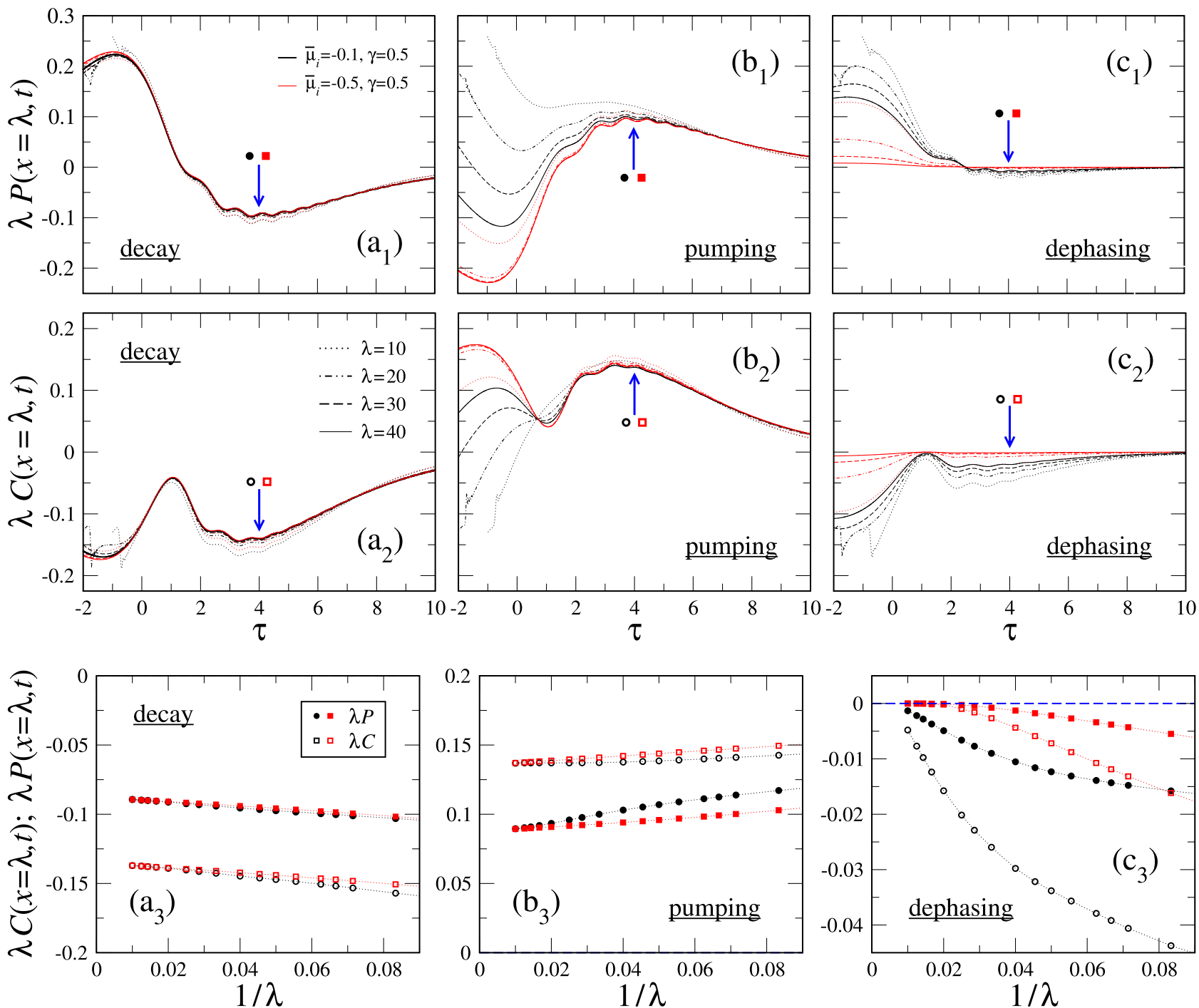

FIG. 4. Same kind of analysis as in Fig. 3 , but fixing the initial Hamiltonian parameter $\bar{\mu}_{i}$, rather than the initial time $\tau_{i}$. We again show results for $x / \lambda=1$. In all the panels we kept the rescaled dissipation strength fixed and equal to $\gamma=0.5$. The color code refers to $\bar{\mu}_{i}=-0.1$ (black) and to $\bar{\mu}_{i}=-0.5$ (red), while different line styles stand for various values of the length scale $\lambda$, from 10 to 40 . [( $\left.\left.a_{3}\right),\left(b_{3}\right),\left(c_{3}\right)\right]$ Rescaled correlations $\lambda P(x, t)$ (filled symbols) and $\lambda C(x, t)$ (empty symbols) as a function of $1 / \lambda$, for $\bar{\mu}_{i}=-0.1$ (circles) or $\bar{\mu}_{i}=-0.5$ (squares), at fixed $\tau=4$ (arrows in the above panels). Panels in the three columns refer to incoherent decay $\left[\left(a_{1}\right),\left(a_{2}\right),\left(a_{3}\right)\right]$, pumping $\left[\left(b_{1}\right),\left(b_{2}\right),\left(b_{3}\right)\right]$, and dephasing $\left[\left(c_{1}\right),\left(c_{2}\right),\left(c_{3}\right)\right]$.

tive in destroying this type of correlations: with increasing $\gamma$, the various curves rapidly decay to a very small asymptotic value for $\lambda$ large [i.e., green curves for $\gamma=10$ in Figs. 3( $\left.c_{1}\right)$ and $3\left(c_{2}\right)$ are hardly distinguishable from zero]. On the other hand, for the incoherent decay or pumping, definitely larger values of $\gamma$ are required to suppress correlations.

The convergence to the asymptotic behavior is analyzed more in depth in Figs. 3( $\left.a_{3}\right), 3\left(b_{3}\right)$, and $3\left(c_{3}\right)$ for a fixed $\tau$, where we explicitly show the dependence of the correlation functions on $1 / \lambda$, up to $\lambda=100$. Our data hint at the presence of $1 / \lambda$ power-law corrections, similarly to what has been observed for the unitary case (compare with Fig. 1).

Even in the presence of dissipation, the dynamic KZ scaling should not depend on the choice of the initial $\bar{\mu}_{i}$, if this is kept fixed, and thus one expects the scaling behavior reported in Eqs. (24a)-(24b). This has been verified numerically by fixing $\bar{\mu}_{i}<0$, as shown in Fig. 4 for $P(x, t)$ [Figs. 4(a $\left.\mathrm{a}_{1}\right), 4\left(\mathrm{~b}_{1}\right)$, and $4\left(\mathrm{c}_{1}\right)$ ] and $C(x, t)$ [Figs. $4\left(\mathrm{a}_{2}\right), 4\left(\mathrm{~b}_{2}\right)$, and $\left.4\left(\mathrm{c}_{2}\right)\right]$, and in Fig. 5 for $G(x, t)$ [Figs. $5\left(\mathrm{a}_{1}\right)$ and $5\left(\mathrm{~b}_{1}\right)$ ]. The displayed data are for a specific value of $\gamma=0.5$ and for two different values of $\bar{\mu}_{i}=-0.1$ (black) and -0.5 (red). The various curves stand for different values of $\lambda$. In all cases we observe that they approach the same asymptotic behavior, irrespective of the choice of $\bar{\mu}_{i}$; the approach becomes faster, when further increasing $\left|\bar{\mu}_{i}\right|$ (not shown). Note that, for the incoherent decay, this appears to be much faster than for the other kinds of dissipation (especially for $\mathrm{KZ}$ protocols with $\bar{\mu}_{i}=-0.1$, starting close to the critical point $\bar{\mu}=0$ ). In contrast, as suggested in Figs. 3( $\left.\mathrm{c}_{1}\right)$ and $3\left(\mathrm{c}_{2}\right)$ for fixed $\tau_{i}$ and different $\gamma$, dephasing seems to be the most disruptive dissipation mechanism: the red curves in Figs. 4(c $\left.c_{1}\right)$ and 4(c $\left.c_{2}\right)$ (where $\left|\tau_{i}\right|$ is much larger than for the black ones) are rapidly suppressed with $\lambda$. 

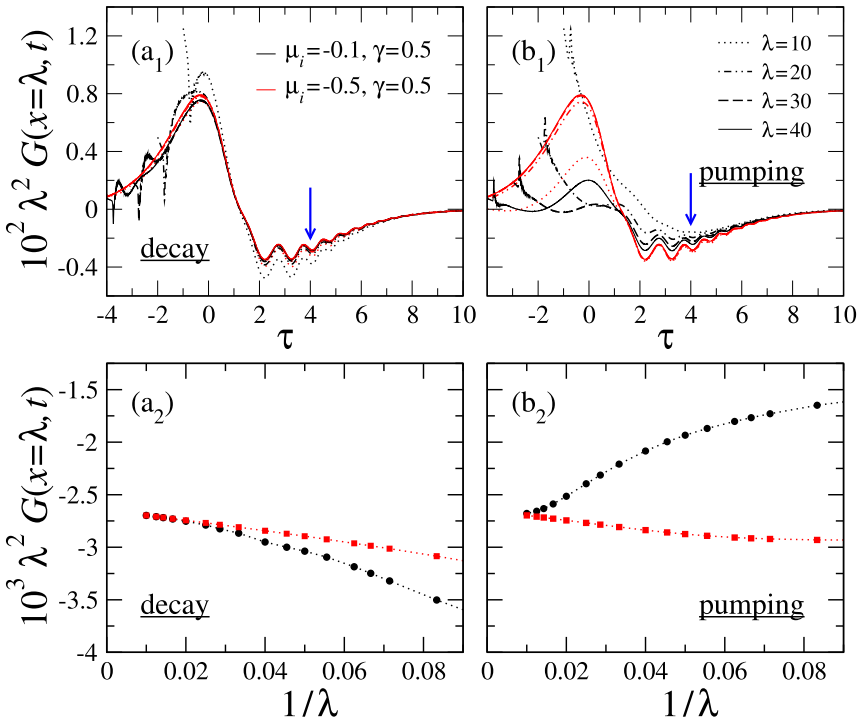

FIG. 5. Same analysis as in Fig. 4, but for the rescaled correlation $\lambda^{2} G(x, t)$. We have computed it the presence of either incoherent decay or pumping, since with dephasing we were only able to compute two-point observables (while density-density correlations are a four-point observable) [8].

Figures $4\left(a_{3}\right), 4\left(b_{3}\right), 4\left(c_{3}\right), 5\left(a_{2}\right)$, and $5\left(b_{2}\right)$ show the convergence to the asymptotic behavior with $\lambda$ and for a given $\tau$, which is again expected to be a power law. Note that the speed with $\lambda$ at which data for the two $\bar{\mu}_{i}$ converge to the same value depends on the type of dissipation and observable. In general, we observe that for decay the convergence is much faster than in the other cases. Moreover, in the limit $\lambda \rightarrow \infty$, while with either decay or pumping the correlators go toward a nonzero value, with dephasing the scaling functions (for $\tau$ sufficiently larger than zero) are compatible with zero.

We conclude this part by emphasizing that, in order to obtain the correct $\mathrm{KZ}$ asymptotic scaling behavior, it is crucial to allow for the dissipation to be suitably rescaled, so that it asymptotically approaches the zero value in the $t_{s} \rightarrow \infty$ limit. That is, the parameter $u$ entering the dissipator in the Lindblad master equation (4) must be scaled as $u \sim t_{s}^{-\kappa}$, with $\kappa=z /\left(y_{\mu}+z\right)$, meaning that the scaling variable $\gamma=u t_{s}^{\kappa}$ has to be kept fixed [see Eq. (23)]. In contrast, if the dissipation strength $u$ is not changed with $t_{s}$, one would obtain the result of Fig. 6, where we plot the rescaled correlation $\lambda P(x, t)$ as a function of $\tau$, fixing all the required scaling variables except that associated to the incoherent particle loss mechanism $(\gamma)$. We note that, contrary to all the cases studied before, here the various curves for different $\lambda$ values do not approach a scaling behavior in the $\lambda=\sqrt{t_{s}} \rightarrow \infty$ limit, but rather seem to converge to a trivial regime dominated by the dissipation, where all the long-distance correlations drop to zero.

\section{B. Dynamic KZ finite-size scaling}

We now switch to systems with finite size, and utilize the FSS framework of Sec. IV C to analyze the behavior of the dissipative Kitaev wire undergoing a $\mathrm{KZ}$ protocol which crosses the quantum transition point. Results for the three

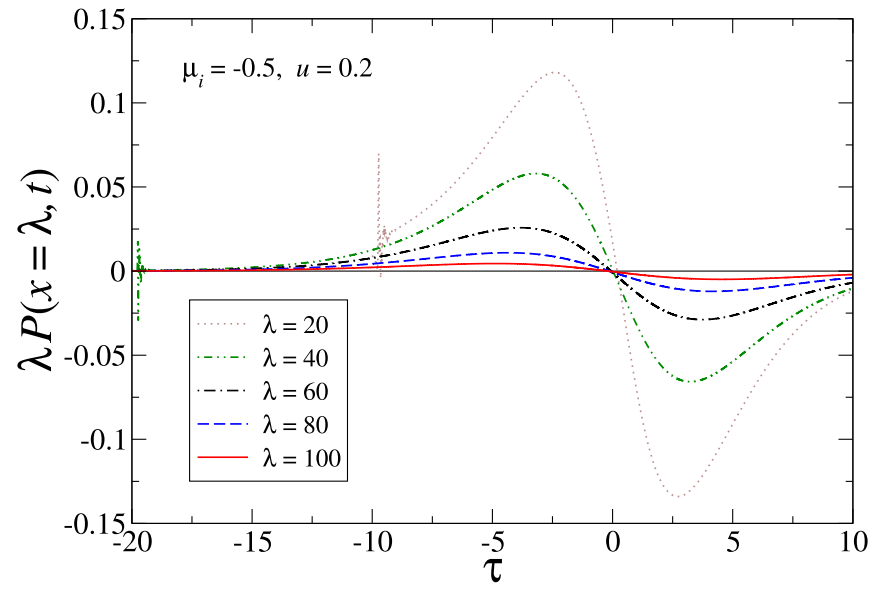

FIG. 6. Rescaled correlation $\lambda P(x, t)$, fixing $x / \lambda=1$ and $\bar{\mu}_{i}=-0.5$, for the dissipative Kitaev quantum wire in the thermodynamic limit, as a function of the scaling time variable $\tau$. The dissipation has been chosen in the form of incoherent particle losses, with a coupling $u$ entering the Lindblad master equation (4) that is kept fixed and equal to $u=0.2$. Different lines stand for various values of the length scale $\lambda$, from 20 to 100 (see legend). Note that the various curves start from different initial rescaled times $\tau_{i}=\lambda \bar{\mu}_{i}$.

fixed-time correlation functions $P(x, t), C(x, t)$, and $G(x, t)$ are reported in Fig. 7, where we analyze their temporal behavior along a $\mathrm{KZ}$ protocol associated with a slow variation of the Hamiltonian parameter $\bar{\mu}$ from negative to positive values, in the presence of incoherent particle losses. Following the FSS scaling behavior of Eq. (26), we kept fixed the ratio $x / L$, the parameter $v$ inversely proportional to the speed of the driving [cf. Eq. (19)], the rescaled dissipation strength $\gamma_{L}$ [cf. Eq. (27)], and the initial rescaled time $\tau_{i}<0$. Note that, in the FSS framework, the dissipation strength $u=\gamma_{L} / L$ entering the master equation (4) is inversely proportional to the system size, thus scaling down to zero in the limit $L \rightarrow \infty$.

In all cases, the rescaled correlations nicely approach a scaling function with increasing $L$, as predicted by the scaling law in Eq. (26). The finite-size approach to the asymptotic behavior is compatible with a $L^{-1}$ behavior, as highlighted in the inset of Fig. 7(c) at fixed $\tau$. We also observe that, for small values of $\tau$, the scaling curves develop complex nonanalytic spikes in $\tau$, the magnitude and frequency of which increase with $L$, similarly to other dynamic situations such as after sudden quenches [8,9]; for larger $\tau$ dissipation tends to smear those apparent singularities.

To shed light on the effects of the system-bath coupling, in Fig. 8 we have analyzed the correlation $C(x, t)$ for the three different types of dissipation of Eq. (31), and for varying rescaled strength $\gamma_{L}$ as indicated in the legends. We have also reported the KZ behavior in the unitary case (black curves), to be compared with that in the presence of an environmental interaction (colored curves). Besides the nice convergence to a scaling function for $L \rightarrow \infty$, we observe that dephasing appears to be more effective in destroying correlations, since, with increasing $\gamma_{L}$, the curves rapidly approach the zero value in time; in contrast, for both incoherent decay and incoherent pumping, definitely larger values of $\gamma_{L}$ are required to suppress correlations. Moreover, in the presence of pumping, 

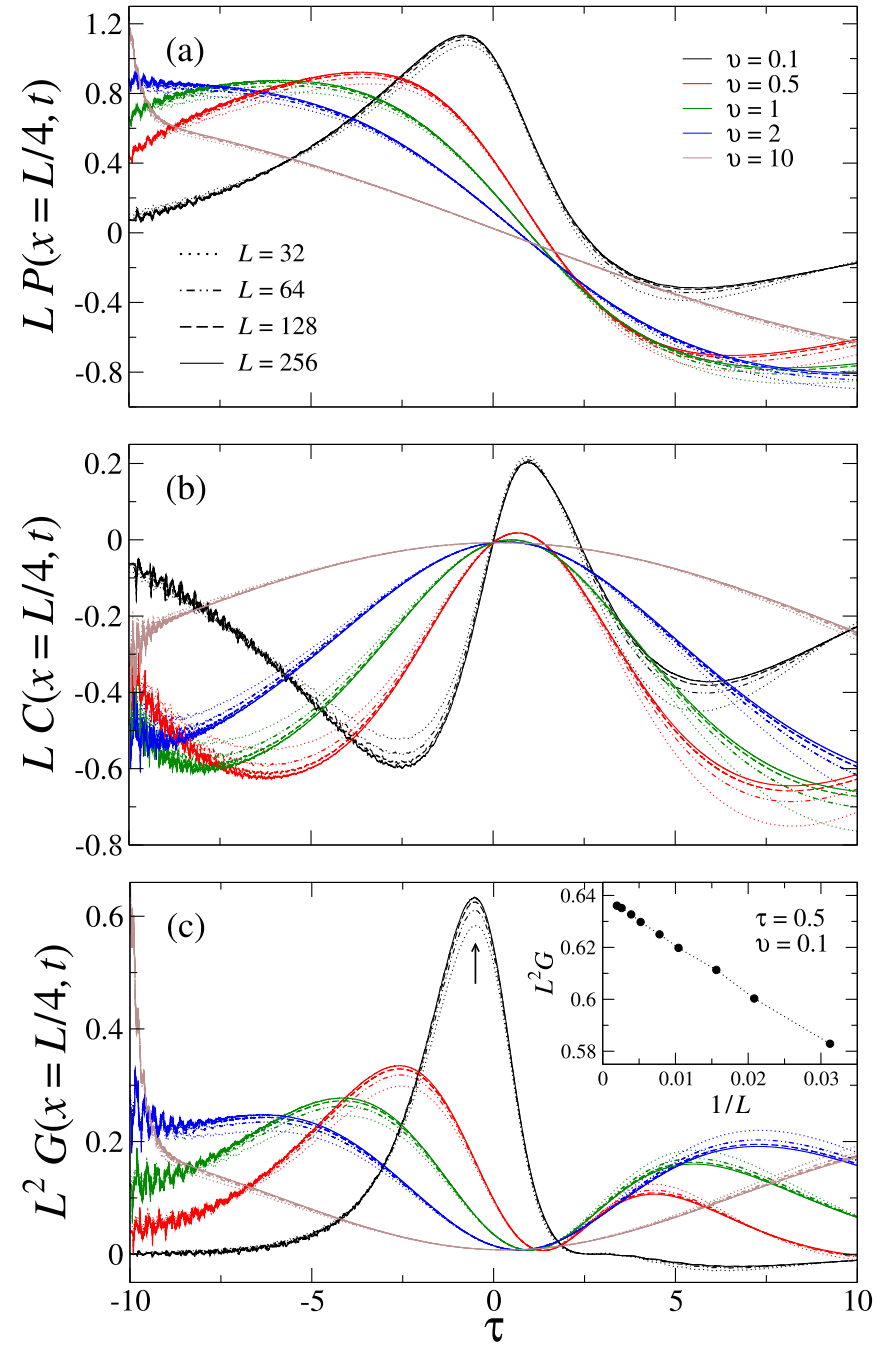

FIG. 7. Rescaled correlations $L P(x, t)$ (a), $L C(x, t)$ (b), and $L^{2} G(x, t)$ (c), fixing $x / L=1 / 4$ (results for other values of $x / L$ show analogous behaviors), for the dissipative Kitaev quantum wire with a finite length $L$, as a function of the scaling variable $\tau$. The color code corresponds to several values of the inverse $\mathrm{KZ}$ speed $v$, while different line styles stand for various system sizes $L$ (see legends). Here we fix the scaling variables associated to the initial time $\left(\tau_{i}=-10\right)$ and to the dissipation $\left(\gamma_{L}=1\right)$, which has been chosen in the form of incoherent particle losses. The inset in panel (c) displays the rescaled correlation $L^{2} G(x, t)$ as a function of $1 / L$ (data up to $L=512$ ), for fixed $v=0.1$ and $\tau=-0.5$ (arrow in the main panel).

even a tiny amount of dissipation is capable to drive the system far from the equilibrium state in the $\tau<0$ side (see also the discussion in Sec. VI A 2).

We have also numerically verified that, analogously to the dynamic scaling behavior in the infinite-volume limit, the dynamic FSS functions do not depend on the initial Hamiltonian parameter $\bar{\mu}_{i}<0$ if this is kept fixed in the dynamic KZ limit [see Eq. (28)]. Figure 9 reports the behavior of the rescaled correlation $L P(x, t)$ as a function of $\tau$, for three different choices of $\bar{\mu}_{i}$, and for dissipation provided by incoherent decay at fixed $\gamma_{L}$. Figures $9\left(a_{1}\right), 9\left(b_{1}\right)$, and $9\left(c_{1}\right)$ show convergence with $L$ to a scaling function, which appears

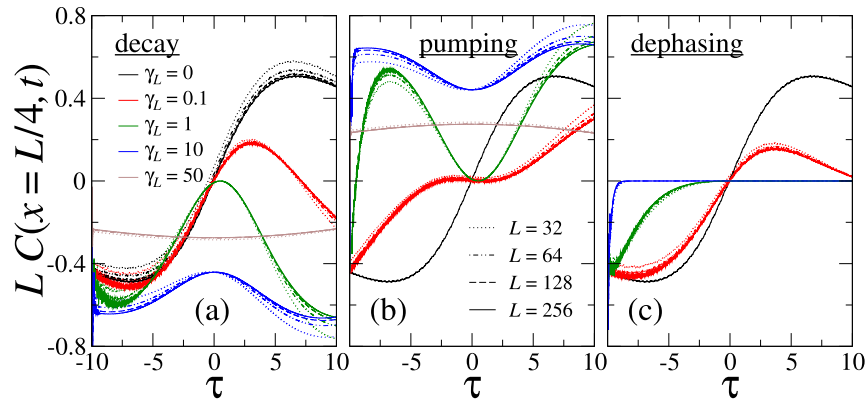

FIG. 8. Same as in Fig. 7, but for the correlation $C(x, t)$, for dissipation given by decay (a), pumping (b), or dephasing (c). The color code corresponds to several values of $\gamma_{L}$, while different line styles stand for various values of $L$ (see legends). Black curves are for $\gamma_{L}=0$ [in panels (b) and (c) we replot the same curve corresponding to $L=256$, for reference]. Here we fix $\tau_{i}=-10$ and $v=1$.

to be the same: Figs. $9\left(a_{2}\right), 9\left(b_{2}\right)$, and $9\left(c_{2}\right)$ unveil how tiny discrepancies in the temporal behavior, starting from different $\bar{\mu}_{i}$, can be suppressed in the large- $L$ limit.

\section{SUMMARY AND CONCLUSIONS}

We have investigated the interplay between coherent and dissipative drivings in the dynamics of quantum many-body systems subject to $\mathrm{KZ}$ protocols across continuous quantum transitions, starting from the gapped disordered phase, that is, when one Hamiltonian parameter is slowly driven across its critical value, for example, with a linear dependence on time. Specifically, the issue we have addressed here is whether, and
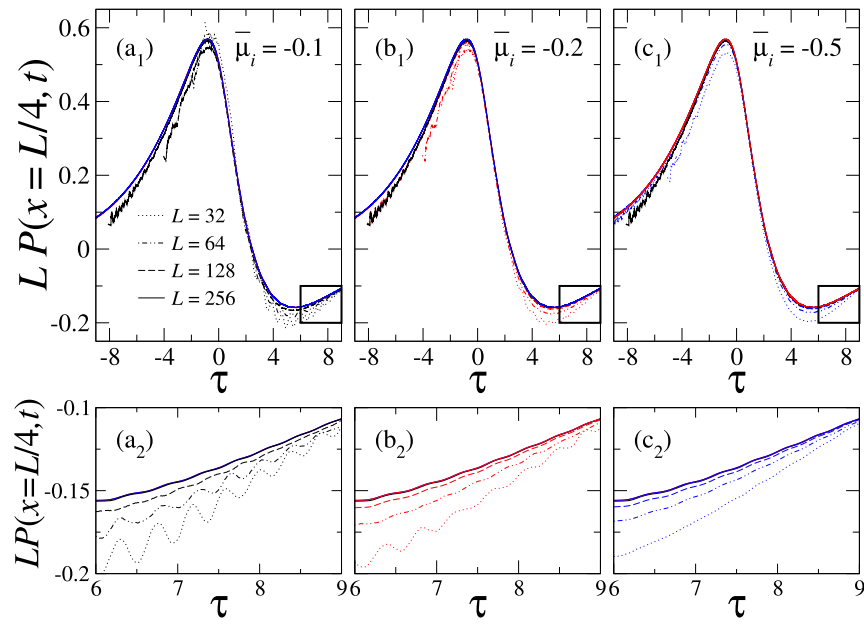

FIG. 9. The rescaled correlation $L P(x, t)$ with $x / L=1 / 4$ as a function of $\tau$ (results for other values of $x / L$ show analogous behaviors), fixing the initial Hamiltonian parameter $\bar{\mu}_{i}=-0.1\left[\left(\mathrm{a}_{1}\right)\right.$, $\left(\mathrm{a}_{2}\right)$, black curves], $-0.2\left[\left(\mathrm{~b}_{1}\right),\left(\mathrm{b}_{2}\right)\right.$, red curves], and $-0.5\left[\left(\mathrm{c}_{1}\right)\right.$, $\left(c_{2}\right)$, blue curves]. Different line styles stand for various $L$ (see legend). $\left(a_{2}\right),\left(b_{2}\right),\left(c_{2}\right)$ Magnifications of panels $\left(a_{1}\right),\left(b_{1}\right)$, and $\left(c_{1}\right)$, for $6 \leqslant \tau \leqslant 9$. Here we fix $v=0.1$, and $\gamma_{L}=1$ with dissipation given by incoherent decay. Note that the three continuous curves, corresponding to the largest size $L=256$ and different $\bar{\mu}_{i}$, are plotted in each of the six panels and cannot be distinguished on the scale of the plots reported. 
under which conditions, open dissipative systems can develop a universal dynamic scaling regime similar to that shown by closed systems at quantum transitions, characterized by asymptotic homogeneous scaling laws. To this purpose we have focused on a class of dissipative mechanisms, the dynamics of which can be reliably described through a Lindblad master equation governing the time evolution of the density matrix of the open system.

The perturbation arising from the dissipation turns out to be relevant at the quantum transition $[8,9]$. This implies that open systems cannot develop asymptotic dynamic scaling behaviors controlled by the universality class of the quantum transition when keeping the dissipation decay rate $u$ finite and fixed in the critical limit of the Hamiltonian parameter. This is analogous to the effect of any relevant RG perturbation close to a critical point [7], like the temperature, which makes the system run away from criticality. Nevertheless, we argue that a dynamic $\mathrm{KZ}$ scaling limit exists in the presence of a sufficiently weak dissipation. Such a scaling limit, controlled by the universality class of the quantum transition, arises in a regime of weak dissipation. Indeed, it requires a tuning of the dissipative interactions, and in particular of the decay-rate parameter $u$ of the Lindblad master equation describing the evolution of the density matrix [see Eq. (4)]. The decay rate $u$ must decrease as $u \sim t_{s}^{-\kappa}$ when increasing the time scale $t_{s}$ of the $\mathrm{KZ}$ protocol, where the positive exponent $\kappa=z /\left(y_{\mu}+z\right)<1$ depends on the dynamic exponent $z$ and the RG dimension $y_{\mu}$ of the driving Hamiltonian parameter (usually related to the correlation-length exponent $v$ by $\left.v=y_{\mu}^{-1}\right)$. The resulting dynamic KZ scaling laws, allowing for the presence of dissipation, provide a unique framework to discuss the interplay between (critical) coherent and dissipative drivings.

The dynamic KZ scaling scenario has been checked within fermionic wires [see Eq. (29)], in the presence of homogeneous dissipation due to local incoherent pumping, decay, and dephasing, which are described by the Lindblad operators reported in Eq. (31). The particularly convenient choice of this model enables us to scale its complexity linearly with its size, allowing us to simulate the exact dissipative dynamics of systems with thousands of sites; we have thus elected it as a testbed for accurate numerical investigations of the many-body Lindblad master equation. Our numerical analysis ultimately supports the phenomenological dynamic KZ scaling framework addressing the competition between coherent dynamics and dissipation at a continuous quantum transition.

We believe that, in the near future, it will be also possible to address and verify this scenario through suitably engineered experiments with ultracold atoms or cavity-QED technology aimed at realizing and controlling driven-dissipative quantum many-body systems (see, e.g., Ref. [70]).

It would be tempting to investigate and carefully verify our dynamic KZ scaling in other quantum dissipative systems, such as Ising-like quantum spin models. To that purpose, given the difficulties in finding a numerical solution to the Lindblad master equation for a generic many-body problem (4), a FSS framework should be adopted as the primary setting, due to the relatively small system sizes that could be reached and the substantial impossibility to address infinitevolume systems (this would be the case, e.g., for the standard quantum Ising chain, with realistic local dissipation related to the spin operators). In that respect, an interesting issue would be to extend the dynamic KZ scaling to protocols across firstorder quantum transitions (e.g., in the quantum Ising chain in a transverse plus longitudinal field). The exponentially closing gap between the two lowest states of the ordered phase might be relevant and new features may become apparent for systems with $O(10)$ spins, such as sensitivity to the type of boundary conditions $[63,71,72]$.

\section{APPENDIX: SIMILARITIES AND DIFFERENCES BETWEEN THE KITAEV WIRE AND THE QUANTUM ISING CHAIN}

In Sec. $\mathrm{V}$ we stated that the Kitaev quantum wire described by the Hamiltonian (29) undergoes a continuous quantum transition in the same universality class of the quantum Ising chain. The similarities between the two models can be put on a formal ground by means of a Jordan-Wigner transformation, which maps the spinless fermions into spin-1/2 operators:

$$
\hat{\sigma}_{j}^{ \pm}=\exp \left(i \pi \sum_{\ell<j} \hat{n}_{\ell}\right) \hat{c}_{j}^{ \pm} .
$$

Here $\hat{\sigma}_{j}^{ \pm}=\frac{1}{2}\left(\hat{\sigma}_{j}^{x} \pm i \hat{\sigma}_{j}^{y}\right)$ are the spin-1/2 raising/lowering operators and $\hat{\sigma}_{j}^{\alpha}(\alpha=x, y, z)$ denote the usual Pauli matrices associated to site $j$ in the chain.

Indeed, it can be easily shown that, neglecting boundary terms, the above transformation maps $\hat{H}_{\mathrm{K}}$ of Eq. (29) into the $X Y$ chain $(\delta \neq 0)$ :

$$
\hat{H}_{X Y}=-t \sum_{j}\left[\frac{1+\delta}{2} \hat{\sigma}_{j}^{x} \hat{\sigma}_{j+1}^{x}+\frac{1-\delta}{2} \hat{\sigma}_{j}^{y} \hat{\sigma}_{j+1}^{y}+\frac{\mu}{2 t} \hat{\sigma}_{j}^{z}\right] .
$$

In particular, for $t=\delta=1$, the corresponding spin model coincides with the quantum Ising chain

$$
\hat{H}_{\mathrm{Is}}=-\sum_{j}\left(\hat{\sigma}_{j}^{x} \hat{\sigma}_{j+1}^{x}+g \hat{\sigma}_{j}^{z}\right),
$$

with $g=-\mu / 2$.

It is, however, crucial to stress that the boundary conditions play an important role in this mapping. As a matter of fact, the nonlocal Jordan-Wigner transformation of the Ising chain with periodic or antiperiodic boundary conditions does not map into the fermionic model (29) with periodic or antiperiodic boundary conditions. Indeed further considerations apply $[73,74]$, leading to a less straightforward correspondence, which also depends on the parity of the particle number eigenvalue (see below). Therefore, although the bulk behaviors of the above models in the infinite-volume limit (and thus their phase diagram) are analogous, the resulting FSS functions are different, since they subtly depend on the choice of the boundary conditions.

Even more, the Kitaev quantum wire with antiperiodic boundary conditions, explicitly studied in this paper, turns out to be gapped in both of the phases separated by the quantum transition at $\mu_{c}=-2$. Indeed, the energy difference $\Delta$ of the two lowest states is given by

$$
\Delta=\sqrt{\bar{\mu}^{2}+4(2-\bar{\mu})[1-\cos (\pi / L)]},
$$


where $\bar{\mu}=\mu-\mu_{c}$, such that

$$
\Delta=\left\{\begin{array}{lll}
|\bar{\mu}|+\frac{\pi^{2}(2-\bar{\mu})}{|\bar{\mu}| L^{2}}+O\left(L^{-4}\right) & \text { for } & |\bar{\mu}|>0, \\
\frac{2 \pi}{L}+O\left(L^{-3}\right) & \text { for } & |\bar{\mu}|=0 .
\end{array}\right.
$$

Therefore, the Kitaev quantum wire studied here does not exhibit the lowest-state degeneracy of the ordered phase of the quantum Ising chain (i.e., the exponential suppression of the gap with increasing $L$ ). The reason for that substantial discrepancy resides in the fact that the Hilbert space of the Kitaev quantum wire with antiperiodic boundary conditions alone is restricted with respect to that of the quantum Ising chain, so that it is not possible to restore the competition between the two vacua belonging to the symmetric/antisymmetric sectors of the Ising model $[43,62,73]$.

The ultimate reason why we prefer to stick with the Kitaev quantum wire is twofold: (i) the dissipation that we consider in this paper is more naturally defined for Fermi lattice gases, and (ii) the dissipative fermionic decay and pumping mechanisms cannot be mapped into simple spin operators, due to the presence of a nonlocal string operator in the transformation (A1). In this respect, simulating a conventional quantum
Ising chain with local dissipation in the form of spin losses $\left(\hat{L}_{\mathrm{l}, j}=\hat{\sigma}_{j}^{-}\right)$or pumping $\left(\hat{L}_{\mathrm{p}, j}=\hat{\sigma}_{j}^{+}\right)$would prevent one from exploiting the particularly simple solvability of the Kitaev model with a polynomial amount of resources, due to the appearance of Jordan-Wigner strings when mapping the term $\hat{L}_{j} \rho \hat{L}_{j}^{\dagger}$ of the Lindblad master equation in fermionic language [29].

In light of this, it is, finally, worth mentioning that, although we have only shown numerical results for $\mathrm{KZ}$ protocols where the Hamiltonian parameter $\mu$ is linearly driven in time from an initial value $\mu_{i}<\mu_{c}$ to a final value $\mu_{f}>\mu_{c}$, there is no reason to expect qualitative differences when reverting the protocol, i.e., starting from $\mu_{i}>\mu_{c}$ and ending with $\mu_{f}<\mu_{c}$. The reason resides in the fact that the Kitaev chain with antiperiodic boundary conditions is gapped in both phases on the left and on the right of the quantum transition point $\mu_{c}$, and thus the evolution arising from slow changes of $\mu$ is essentially adiabatic, from any $\bar{\mu}_{i}$ far from criticality to the relevant scaling interval around $\mu_{c}$. The situation may change for the above-mentioned quantum Ising chain, since one of the two phases is ordered and presents a double degeneracy (see, e.g., Ref. [75]).
[1] J. I Cirac and P. Zoller, Goals and opportunities in quantum simulation, Nat. Phys. 8, 264 (2012).

[2] I. Bloch, J. Dalibard, and S. Nascimbène, Quantum simulations with ultracold quantum gases, Nat. Phys. 8, 267 (2012).

[3] R. Blatt and C. F. Roos, Quantum simulations with trapped ions, Nat. Phys. 8, 277 (2012).

[4] A. Aspuru-Guzik and P. Walther, Photonic quantum simulators, Nat. Phys. 8, 285 (2012).

[5] A. A. Houck, H. E. Türeci, and J. Koch, On-chip quantum simulation with superconducting circuits, Nat. Phys. 8, 292 (2012).

[6] I. M. Georgescu, S. Ashhab, and F. Nori, Quantum simulation, Rev. Mod. Phys. 86, 153 (2014).

[7] S. Sachdev, Quantum Phase Transitions (Cambridge University, Cambridge, England, 1999).

[8] D. Nigro, D. Rossini, and E. Vicari, Competing coherent and dissipative dynamics close to quantum criticality, Phys. Rev. A 100, 052108 (2019).

[9] D. Rossini and E. Vicari, Scaling behavior of the stationary states arising from dissipation at continuous quantum transitions, Phys. Rev. B 100, 174303 (2019).

[10] T. W. B. Kibble, Topology of cosmic domains and strings, J. Phys. A 9, 1387 (1976).

[11] W. H. Zurek, Cosmological experiments in superfluid Helium? Nature (London) 317, 505 (1985).

[12] W. H. Zurek, U. Dorner, and P. Zoller, Dynamics of a Quantum Phase Transition, Phys. Rev. Lett. 95, 105701 (2005).

[13] A. Polkovnikov and V. Gritsev, Breakdown of the adiabatic limit in low-dimensional gapless systems, Nat. Phys. 4, 477 (2008).

[14] A. Chandran, A. Erez, S. S. Gubser, and S. L. Sondhi, KibbleZurek problem: Universality and the scaling limit, Phys. Rev. B 86, 064304 (2012).
[15] S. Ulm et al., Observation of the Kibble-Zurek scaling law for defect formation in ion crystals, Nat. Commun. 4, 2290 (2013).

[16] K. Pyka et al., Topological defect formation and spontaneous symmetry breaking in ion Coulomb crystals, Nat. Commun. 4, 2291 (2013).

[17] G. Lamporesi, S. Donadello, S. Serafini, F. Dalfovo, and G. Ferrari, Spontaneous creation of Kibble-Zurek solitons in a Bose-Einstein condensate, Nat. Phys. 9, 656 (2013).

[18] M. Gong et al., Simulating the Kibble-Zurek mechanism of the Ising model with a superconducting qubit system, Sci. Rep. 6, 22667 (2016).

[19] M. Anquez, B. A. Robbins, H. M. Bharath, M. Boguslawski, T. M. Hoang, and M. S. Chapman, Quantum Kibble-Zurek Mechanism in a Spin-1 Bose-Einstein Condensate, Phys. Rev. Lett. 116, 155301 (2016).

[20] A. Keesling et al., Quantum Kibble-Zurek mechanism and critical dynamics on a programmable Rydberg simulator, Nature (London) 568, 207 (2019).

[21] J. Dziarmaga, Dynamics of a quantum phase transition and relaxation to a steady state, Adv. Phys. 59, 1063 (2010).

[22] A. Polkovnikov, K. Sengupta, A. Silva, and M. Vengalattore, Colloquium: Nonequilibrium dynamics of closed interacting quantum systems, Rev. Mod. Phys. 83, 863 (2011).

[23] A. Fubini, G. Falci, and A. Osterloh, Robustness of adiabatic passage through a quantum phase transition, New J. Phys. 9, 134 (2007).

[24] D. Patanè, A. Silva, L. Amico, R. Fazio, and G. E. Santoro, Adiabatic Dynamics in Open Quantum Critical Many-Body Systems, Phys. Rev. Lett. 101, 175701 (2008).

[25] D. Patanè, L. Amico, A. Silva, R. Fazio, and G. E. Santoro, Adiabatic dynamics of a quantum critical system coupled to 
an environment: Scaling and kinetic equation approaches, Phys. Rev. B 80, 024302 (2009).

[26] P. Nalbach, S. Vishveshwara, and A. A. Clerk, Quantum KibbleZurek physics in the presence of spatially correlated dissipation, Phys. Rev. B 92, 014306 (2015).

[27] A. Dutta, A. Rahmani, and A. del Campo, Anti-Kibble-Zurek Behavior in Crossing the Quantum Critical Point of a Thermally Isolated System Driven by a Noisy Control Field, Phys. Rev. Lett. 117, 080402 (2016).

[28] Z.-P. Gao, D.-W. Zhang, Y. Yu, and S.-L. Zhu, Anti-KibbleZurek behavior of a noisy transverse-field XY chain and its quantum simulation with two-level systems, Phys. Rev. B 95, 224303 (2017).

[29] M. Keck, S. Montangero, G. E. Santoro, R. Fazio, and D. Rossini, Dissipation in adiabatic quantum computers: lessons from an exactly solvable model, New. J. Phys. 19, 113029 (2017).

[30] V. N. Smelyanskiy, D. Venturelli, A. Perdomo-Ortiz, S. Knysh, and M. I. Dykman, Quantum Annealing via EnvironmentMediated Quantum Diffusion, Phys. Rev. Lett. 118, 066802 (2017).

[31] P. Hedvall and J. Larson, Dynamics of non-equilibrium steady state quantum phase transitions, arXiv:1712.01560.

[32] L. Arceci, S. Barbarino, D. Rossini, and G. E. Santoro, Optimal working point in dissipative quantum annealing, Phys. Rev. B 98, 064307 (2018).

[33] L. P. García-Pintos, D. Tielas, and A. del Campo, Spontaneous Symmetry Breaking Induced by Quantum Monitoring, Phys. Rev. Lett. 123, 090403 (2019).

[34] R. Puebla, A. Smirne, S. F. Huelga, and M. B. Plenio, Universal anti-Kibble-Zurek scaling in fully-connected systems, arXiv:1911.06023.

[35] Z. Fei, N. Freitas, V. Cavina, H. T. Quan, and M. Esposito, Work Statistics across a Quantum Phase Transition, Phys. Rev. Lett. 124, 170603 (2020).

[36] S. M. Griffin, M. Lilienblum, K. T. Delaney, Y. Kumagai, M. Fiebig, and N. A. Spaldin, Scaling Behavior and Beyond Equilibrium in the Hexagonal Manganites, Phys. Rev. X 2, 041022 (2012).

[37] S. Yin, P. Mai, and F. Zhong, Nonequilibrium quantum criticality in open systems: The dissipation rate as an additional indispensable scaling variable, Phys. Rev. B 89, 094108 (2014); S. Yin, C.-Y. Lo, and P. Chen, Scaling behavior of quantum critical relaxation dynamics of a system in a heat bath, ibid. 93, 184301 (2016).

[38] G. Lindblad, On the generators of quantum dynamical semigroups, Commun. Math. Phys. 48, 119 (1976).

[39] V. Gorini, A. Kossakowski, and E. C. G. Sudarshan, Completely positive dynamical semigroups of $\mathrm{N}$-level systems, J. Math. Phys. 17, 821 (1976).

[40] H.-P. Breuer and F. Petruccione, The Theory of Open Quantum Systems (Oxford University, New York, 2002).

[41] Á. Rivas and S. F. Huelga, Open Quantum Systems: An Introduction (Springer, New York, 2012).

[42] L. M. Sieberer, M. Buchhold, and S. Diehl, Keldysh field theory for driven open quantum systems, Rep. Prog. Phys. 79, 096001 (2016).

[43] A. Yu. Kitaev, Unpaired Majorana fermions in quantum wires, Phys. Usp. 44, 131 (2001).
[44] S. Gong, F. Zhong, X. Huang, and S. Fan, Finite-time scaling via linear driving, New J. Phys. 12, 043036 (2010).

[45] A. Pelissetto, D. Rossini, and E. Vicari, Dynamic finite-size scaling after a quench at quantum transitions, Phys. Rev. E 97, 052148 (2018).

[46] A. Pelissetto, D. Rossini, and E. Vicari, Out-of-equilibrium dynamics driven by localized time-dependent perturbations at quantum phase transitions, Phys. Rev. B 97, 094414 (2018).

[47] J. Dziarmaga, Dynamics of a Quantum Phase Transition: Exact Solution of the Quantum Ising Model, Phys. Rev. Lett. 95, 245701 (2005).

[48] S. Deng, G. Ortiz, and L. Viola, Dynamical non-ergodic scaling in continuous finite-order quantum phase transitions, Eur. Phys. Lett. 84, 67008 (2008).

[49] M. Kolodrubetz, B. K. Clark, and D. A. Huse, Nonequilibrium Dynamic Critical Scaling of the Quantum Ising Chain, Phys. Rev. Lett. 109, 015701 (2012).

[50] G. Biroli, in Strongly Interacting Quantum Systems out of Equilibrium, Lecture Notes of the Les Houches Summer School Vol. 99, Aug. 2012, edited by T. Giamarchi, A. J. Millis, O. Parcollet, H. Saleur, and L. F. Cugliandolo (Oxford University, New York, 2016).

[51] A. Francuz, J. Dziarmaga, B. Gardas, and W. H. Zurek, Space and time renormalization in phase transition dynamics, Phys. Rev. B 93, 075134 (2016).

[52] P. Calabrese and J. Cardy, Quantum quenches in $1+1$ dimensional conformal field theories, J. Stat. Mech. (2016) 064003.

[53] E. Vicari, Decoherence dynamics of qubits coupled to systems at quantum transitions, Phys. Rev. A 98, 052127 (2018).

[54] D. Nigro, D. Rossini, and E. Vicari, Scaling properties of work fluctuations after quenches near quantum transitions, J. Stat. Mech. (2019) 023104.

[55] D. Rossini and E. Vicari, Scaling of decoherence and energy flow in interacting quantum spin systems, Phys. Rev. A 99, 052113 (2019).

[56] M. M. Rams, J. Dziarmaga, and W. H. Zurek, Symmetry Breaking Bias and the Dynamics of a Quantum Phase Transition, Phys. Rev. Lett. 123, 130603 (2019).

[57] D. Sadhukhan, A. Sinha, A. Francuz, J. Stefaniak, M. M. Rams, J. Dziarmaga, and W. H. Zurek, Sonic horizons and causality in phase transition dynamics, Phys. Rev. B 101, 144429 (2020).

[58] D. Rossini and E. Vicari, Measurement-induced dynamics of many-body systems at quantum transitions, arXiv:2001.11501.

[59] S. Ducci, P. L. Ramazza, W. Gonzáles-Viñas, and F. T. Arecchi, Order Parameter Fragmentation after a Symmetry-Breaking Transition, Phys. Rev. Lett. 83, 5210 (1999).

[60] R. Monaco, J. Mygind, M. Aaroe, R. J. Rivers, and V. P. Koshelets, Zurek-Kibble Mechanism for the Spontaneous Vortex Formation in $\mathrm{Nb}-\mathrm{Al} / \mathrm{Al}_{\text {ox }} / \mathrm{Nb}$ Josephson Tunnel Junctions: New Theory and Experiment, Phys. Rev. Lett. 96, 180604 (2006).

[61] S. L. Sondhi, S. M. Girvin, J. P. Carini, and D. Shahar, Continuous quantum phase transitions, Rev. Mod. Phys. 69, 315 (1997).

[62] M. Campostrini, A. Pelissetto, and E. Vicari, Finite-size scaling at quantum transitions, Phys. Rev. B 89, 094516 (2014).

[63] M. Campostrini, J. Nespolo, A. Pelissetto, and E. Vicari, FiniteSize Scaling at First-Order Quantum Transitions, Phys. Rev. Lett. 113, 070402 (2014). 
[64] B. Horstmann, J. I. Cirac, and G. Giedke, Noise-driven dynamics and phase transitions in fermionic systems, Phys. Rev. A 87, 012108 (2013).

[65] E. B. Davies, Quantum stochastic processes II, Commun. Math. Phys. 19, 83 (1970); Quantum stochastic processes, 15, 277 (1969).

[66] D. E. Evans, Irreducible Quantum Dynamical Semigroups, Commun. Math. Phys. 54, 293 (1977).

[67] S. G. Schirmer and X. Wang, Stabilizing open quantum systems by Markovian reservoir engineering, Phys. Rev. A 81, 062306 (2010).

[68] D. Nigro, On the uniqueness of the steady-state solution of the Lindblad-Gorini-Kossakowski-Sudarshan equation, J. Stat. Mech. (2019) 043202.

[69] V. Eisler, Crossover between ballistic and diffusive transport: the quantum exclusion process, J. Stat. Mech. (2011) P06007.

[70] T. Tomita, S. Nakajima, I. Danshita, Y. Takasu, and Y. Takahashi, Observation of the Mott insulator to superfluid crossover of a driven-dissipative Bose-Hubbard system, Sci. Adv. 3, e1701513 (2017).

[71] A. Pelissetto, D. Rossini, and E. Vicari, Finite-size scaling at first-order quantum transitions when boundary conditions favor one of the two phases, Phys. Rev. E 98, 032124 (2018).

[72] A. Pelissetto, D. Rossini, and E. Vicari, Scaling properties of the dynamics at first-order quantum transitions when boundary conditions favor one of the two phases, arXiv:2004.08360.

[73] S. Katsura, Statistical mechanics of the anisotropic linear Heisenberg model, Phys. Rev. 127, 1508 (1962).

[74] P. Pfeuty, The one-dimensional Ising model with a transverse field, Ann. Phys. (NY) 57, 79 (1970).

[75] M. Białończyk and B. Damski, Dynamics of longitudinal magnetization in transverse-field quantum Ising model: from symmetry-breaking gap to Kibble-Zurek mechanism, J. Stat. Mech. (2020) 013108. 